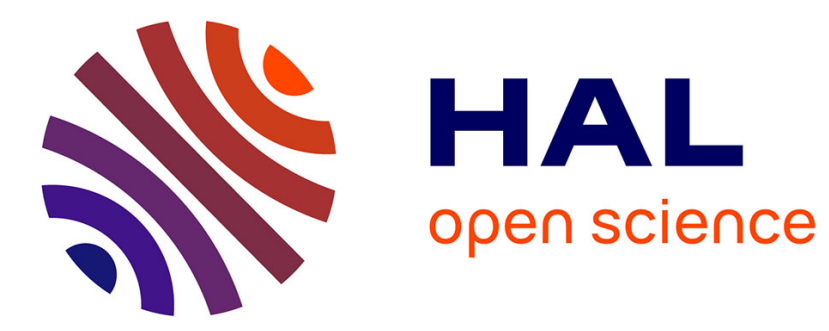

\title{
Comment les grandes familles ottomanes ont découvert la généalogie
}

Olivier Bouquet

\section{To cite this version:}

Olivier Bouquet. Comment les grandes familles ottomanes ont découvert la généalogie. Cahiers de la Méditerranée, 2011. halshs-02136147

\section{HAL Id: halshs-02136147 https://shs.hal.science/halshs-02136147}

Submitted on 21 May 2019

HAL is a multi-disciplinary open access archive for the deposit and dissemination of scientific research documents, whether they are published or not. The documents may come from teaching and research institutions in France or abroad, or from public or private research centers.
L'archive ouverte pluridisciplinaire HAL, est destinée au dépôt et à la diffusion de documents scientifiques de niveau recherche, publiés ou non, émanant des établissements d'enseignement et de recherche français ou étrangers, des laboratoires publics ou privés. 


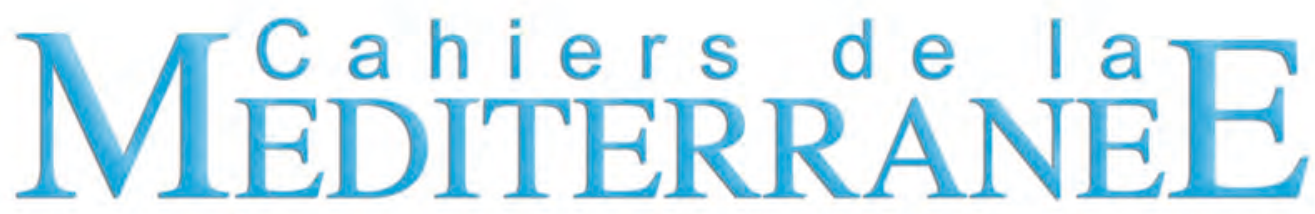

\section{Penser en exil}
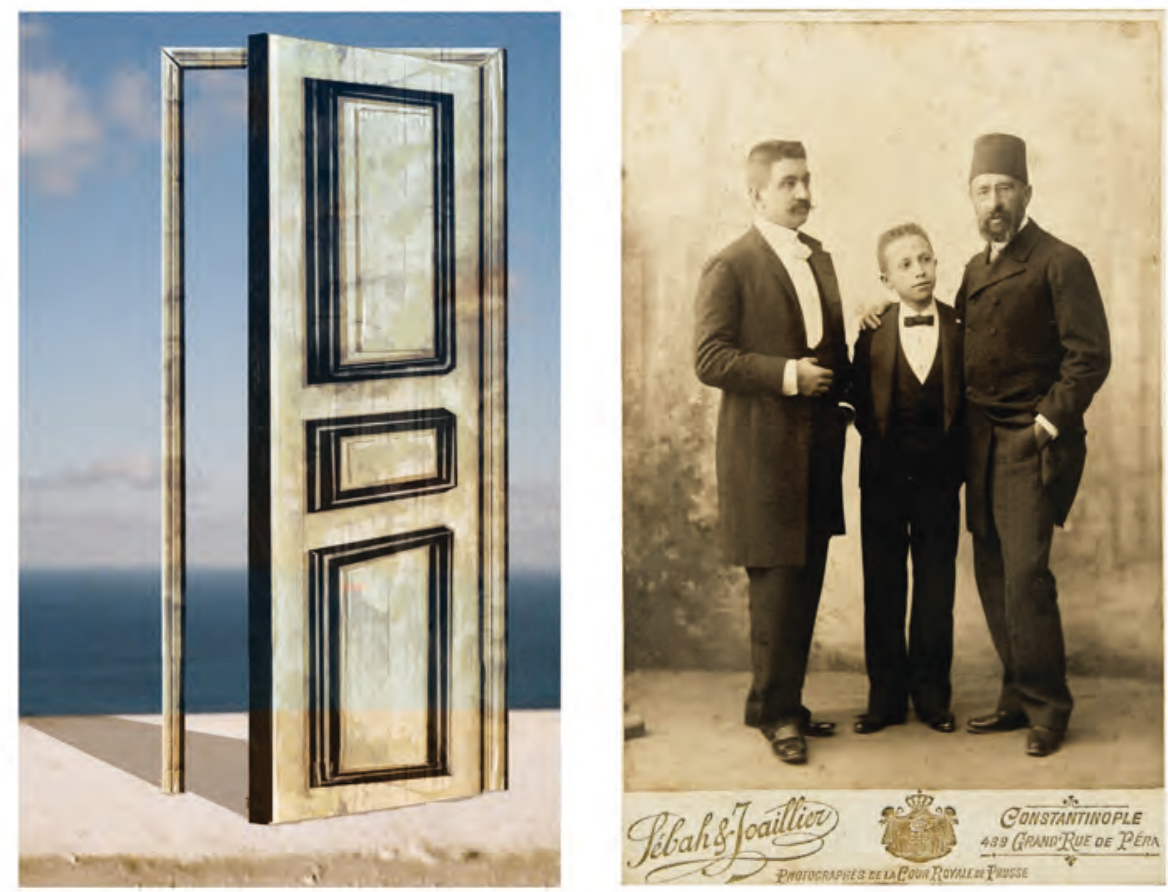

\section{Les grandes familles en Méditerranée orientale}




\title{
Comment les grandes familles ottomanes ont découvert la généalogie ${ }^{1}$
}

\author{
Olivier Bovquet
}

Les Ottomans étaient nombreux à accorder une haute importance aux chaînes de transmission : les familles chérifiennes, en nombre important et croissant au $\mathrm{XVIII}^{\mathrm{e}}$ siècle $^{2}$; les confréries soufies, dépositaires de l'héritage d'un grand maître et garantes de l'authenticité de la filiation initiatique ; les calligraphes, maittres de la culture palatiale, inscrits, génération après génération, dans la lignée de Yakut al-Musta'simi, secrétaire du dernier calife abbasside, al-Musta'sim (I242I258) ; les professeurs de médersa (medrese), soucieux de l'aptitude de leurs élèves à transmettre l'enseignement dispensé ; la famille impériale, honorée pour ses origines connues et inventées, et productrice d'arbres généalogiques à partir de la fin du XVI e siècle ; les grandes lignées d'oulémas, attachées au monopole, acquis de haute lutte, des plus hautes magistratures; les noblesses byzantines et phanariotes, solidaires autour de la défense de leur prestige et la valeur de leurs alliances ; les grandes tribus turkmènes ou kurdes, constituées en dynasties ou quasi-principautés à la veille des réformes du XIX ${ }^{\mathrm{e}}$ siècle. À en croire l'historiographie, ces groupes auraient eu le souci de la généalogie 3 .

Si l'on se place à présent du côté des dignitaires civils et militaires, si l'on considère la période ottomane dans son ensemble, et si l'on fait le point sur les

I. Cet article est le résultat d'un travail de recherche soutenu et financé par l'ANR TRANSTUR (Ordonner et transiger : modalités de gouvernement et d'administration en Turquie et dans l'Empire ottoman, du XIX ${ }^{\mathrm{e}}$ siècle à nos jours). Je tiens à exprimer ma plus vive reconnaissance à Juliette Dumas qui m’a communiqué de précieux documents de vakfyye, à Günsel Renda et Edith Ambros, pour leurs indications bibliographiques, à Faruk Bilici pour ses précisions apportées sur les fondations pieuses, à Edhem Eldem pour ses éclairantes mises au point sur la famille impériale, à Gilles Veinstein, Nicolas Vatin et Nicolas Michel pour leur lecture attentive du texte et leurs corrections, à Catherine Mayeur-Jaouen et Hamit Bozarslan, pour leurs mises en perspective et suggestions comparatives extrêmement stimulantes.

2. Ignatius Mouradgea d'Ohsson dit bien que "presque tous [les seyyid] prétendent avoir des chartes généalogiques, qu'ils appellent Schedjhré ou Silsilé-namé» (on explicitera le sens des termes en italiques plus bas); il les évalue à "au moins la trentième partie de la nation", proportion sans doute largement exagérée (Ignatius Mouradgea d'Ohsson, Tableau général de l'Empire ottoman, tome 4, Istanbul, Isis, 200 (rééd.), p. 310).

3. On trouvera un état des lieux de l'historiographie généalogique ottomane dans Hans Georg Majer, Vorstudien zur Geschichte der Ilmiye im Osmanischen Reich I : Zu Uşakîzade, seiner Familie und seinem Zeyl-i Şakayık, Munich, Rudolph Trofenik, 1978, p. I07-I24. J'y apporte des compléments dans la suite des notes. 
sources dont nous disposons (sans attendre que les papiers de famille révèlent un jour des secrets inespérés), on observe une réalité singulièrement différente. Les grandes familles distinguées par le service du sultan avaient conscience de leur statut et cultivaient leur distinction ; elles formaient une "noblesse dans l'État»" qui n'ignorait pas l'importance des chaînes de transmission et la réalité des solidarités familiales. Il semble pourtant qu'elles ne se constituaient des arbres qu'afin de transmettre des biens ou distribuer des revenus, en particulier dans le cadre de fondations pieuses. C'est la première hypothèse que cet article entend étayer : au sein de familles ottomanes pourtant prestigieuses, la généalogie était un outil gestionnaire au sein de la fondation pieuse $(v a k \imath f)$, avant d'être un référent identitaire ; une infrastructure juridique et économique de la mémoire, avant d'être un appui symbolique du sentiment de la familles. Ce sentiment existait, c'est certain, marqué par une forme d'embourgeoisement de la sphère domestique ; mais ce qui le nourrissait hors des nécessités de la transmission matérielle ne suffisait pas à constituer un genre généalogique ottoman. Le changement eut lieu plus tard, au $\mathrm{xx}^{\mathrm{e}}$ siècle - c'est l'objet d'une seconde hypothèse. C'est parce que les grandes familles de dignitaires furent dépossédées de leur statut et de leur pouvoir qu'elles se constituèrent des arbres et des tableaux ; c'est sous la République turque qu'elles donnèrent au genre généalogique les lettres de noblesse qui ne lui avaient jamais été reconnues dans un État impérial peu enclin à admettre l'existence du fait nobiliaire. Mais dans les cas (plus fréquents qu'on a pu le penser) où les fondations pieuses avaient subsisté et continuaient de produire des bénéfices, ce nouveau goût pour la généalogie - produit complexe d'ostracisme officiel et de culture d'exil - était d'autant plus fort qu'il s'articulait à un intérêt bien compris pour les modalités de transmission des ressources.

Pour bien analyser ce rapport (discret, chronologiquement décalé, et a priori paradoxal) des grandes familles à la mise en forme généalogique, à l'aide de documents d'archives publics (une vakfye ${ }^{6}$ ) et privés (un arbre constitué par une famille), il me faut au préalable faire le point sur l'historiographie ottomaniste. Je commencerai donc par inventorier les représentations généalogiques auxquelles les Ottomans recourraient. Je m'appuierai principalement sur le cas bien documenté de la dynastie impériale, soucieuse de ses origines autant que du bon ordre des successions, mais représentée par ses sultans bien plus que par les membres de la famille impériale. De l'Ottoman aux Ottomans, du Grand Seigneur à ses serviteurs, je déplacerai ensuite l'analyse vers les généalogies de familles de dignitaires, observées à la lumière des conceptions de la noblesse et des formes de représen-

4. Olivier Bouquet, Les Pachas du sultan. Essai sur les agents supérieurs de l'État ottoman (I839-1909), Louvain, Paris, Peeters, 2007, p. 2I2-2I4.

5. Expression empruntée à Philippe Ariès, L'enfant et la vie familiale sous l'Ancien Régime, Paris, Le Seuil, 1973 (rééd.), p. 302. Sur les formes ottomanes de ce sentiment, voir Cem Behar et Alan Duben, Istanbul Households. Marriage, Family and Fertility. I880-1940, Cambridge, Cambridge University Press, 1991 ; Edhem Eldem, "Ottoman Crisis of Legitimacy in the second Half of the Nineteenth Century : A Dynastic Vision ", intervention au colloque Authority and Legitimacy in the Ottoman Empire, Université du Bosphore, Istanbul, 6-7 juillet $200 \mathrm{I}$.

6. On appelle ainsi l'acte juridique par lequel le fondateur définit la vocation du vakrf. 
tation de soi. Au terme de ce parcours, l'horizon sera suffisamment déblayé pour introduire un document généalogique particulier : un diagramme conçu par une famille comme outil de redistribution des ressources d'une fondation pieuse, que j'analyserai à la lumière de la vakfiye à laquelle il se rapportait. J'examinerai alors les deux hypothèses proposées : l'arbre généalogique ottoman comme instrument de solidarité patrimoniale plutôt que comme appui référentiel et symbolique du sentiment de la famille; le genre généalogique comme produit d'une noblesse ostracisée sous la République turque.

\section{Généalogies ottomanes}

\section{Silsilenâme, şecere, soyağacı : teminologie généalogique}

Pour constituer leur propre espace de désignation généalogique, les Ottomans avaient procédé comme à leur habitude : ils avaient emprunté et adapté des notions anciennes. Ils employaient notamment l'expression silsilenâme : composée du terme arabe silsila (chaîne, série) et du persan nâme (souvent utilisé pour désigner des documents écrits ${ }^{7}$ ), celle-ci recouvrait des réalités précises, tantôt distinctes, tantôt combinées. La première était une chaîne de noms, c'est-à-dire nasab, suite patrilinéaire ${ }^{8}$ La seconde désignait une ascendance spirituelle, destinée à relier le chef d'une confrérie à un personnage censé être le fondateur de l'ordre, voire le Prophète. La chaîne était considérée comme essentielle, car elle garantissait l'authenticité de la filiation initiatique?. Il s'agissait d'une sorte d'isnâd ("chaîne de témoignage fondamentale»), conçu selon la base-modèle des transmissions, à savoir le hadîth. Les isnâd du Coran n'existant pas, ceux du hadìth avaient été repris par les cheikhs qui étaient souvent des muhaddithûn (savants chargés de les recueillir et de les transmettre). Cela dit, les isnâd confrériques étaient partiellement charnels, partiellement spirituels, et souvent superposés, le père étant aussi le maître du fils-disciple : la silsila était aussi nasab ${ }^{\text {10 }}$. Le troisième sens était généalogique, c'est-à-dire associé à une dynastie, une maison,

7. «Written pedigree» en est l'une des traductions proposées par Sir James W. Redhouse, $A$ Turkish and English Lexicon, Beyrouth, Librairie du Liban, 1996 (rééd.), p. I 072.

8. Pl. ansab (arabe), ensab (ottoman) : "chaîne, série, suite» (Diran Kélékian, Dictionnaire turcfrançais, Istanbul, Mihran, I9II, p. 689) ; le terme apparaît chez Aşıkpaşazâde cité par Paul Witteck, «Der Stammbaum der Osmanen", Der Islam, vol. I4, I924, p. 94-IOO, p. 95.

9. Citons l'exemple de la silsile descendante de Yunus ("Yunus'un Tarikat silsilesi») : une lignée, ininterrompue, part du prophète Muhammed et passe par Hacı Bektaş ; une autre lignée part également du Prophète, mais est interrompue (signalées en pointillés) (Abdülbâkî Gölpınarlı, Yunus Emre. Hayatr, Istanbul, Bozkurt basımevi, 1936, p. 328). Martin van Bruinessen cite également des cas de lignées interrompues ("The Qadiriyya and the Lineages of Qadiri Shaykhs in Kurdistan ", dans Martin van Bruinessen, Mullas, Sufis and Heretics: The Role of Religion in Kurdish Society, Istanbul, Isis, 2000, p. 216).

IO. Catherine Mayeur-Jaouen, "Le Saint musulman en père de famille», dans Nelly Amri et Denis Gril (dir.), Saint et sainteté. Le regard des sciences de l'homme, Paris, Maisonneuve et Larose MMSH, 2008, p. 249-267. On trouvera divers exemples de silsile de tarikat dans Sadık Vicdâni, Tarikatler ve silsileleri (Tomâr-i Turûk-i Aliyye), ed. Irfan Gündüz, Istanbul, Enderun, 1995. 
une famille chérifienne souvent ${ }^{\mathrm{II}}$. D'un point de vue formel, le silsilenâme pouvait aussi bien renvoyer à une simple «liste généalogique " ${ }^{12}$, un "livre de généalogie $»^{13}$, une "carte généalogique $»^{14}$, ou une «table généalogique $»^{{ }^{15}}$. Et quand on le traduisait par "arbre», souvent il n'en avait pas la forme, à l'instar des arbres occidentaux de la période moderne ${ }^{16}$.

Il en allait différemment du terme şecerenâme, plus directement lié à l'arbre en tant que tel : dérivés de l'arabe šajara (arbre), les termes turcs şecer et şecere avaient gardé ce sens ${ }^{17}$. Le şecerenâme en avait rarement la forme cependant, figurant souvent sur une seule feuille, enroulée, selon une disposition verticale ou horizontale, une forme très "schématique" note Pakal $1 n^{18}$ : généralement une

II. Sir James W. Redhouse, A Turkish..., op. cit., p. I 072.

I2. Ibid., p. I 072 ; Martin van Bruinessen, "The Qadiriyya...", art. cit., p. 2I6.

I3. Charles A. C. Barbier de Meynard, Dictionnaire turc-français, vol. 2, Paris, Ernest Leroux, I886, p. 93.

I4. Diran Kélékian, Dictionnaire..., op. cit., p. 689.

15. Nesep cetveli (Ahmed Vefik Paşa, Lehce-i Osmânî, Ankara, TDK Yay., 200o, p. 8I4).

16. Sur les formes de l'arbre généalogique occidental : Christiane Klapisch-Zuber, «La genèse de l'arbre généalogique", Cahiers du Léopard d'Or, n 2, 1993, p. 4I-8I. Sur les étapes de sa genèse : Christiane Klapisch-Zuber, L'Ombre des ancêtres. Essai sur l'imaginaire médiéval de la parenté, Paris, Fayard, 200o. Sur le lien entre le diagramme généalogique (notamment construit par les spécialistes et anthropologues au $\mathrm{Xx}^{\mathrm{e}}$ siècle) et les métaphores de l'arbre : Mary Bouquet, «Family Trees and their affinities: The Visual imperative of the genealogical diagram ", Journal of the Royal Anthropological Institute of London, vol. 2, $\mathrm{n}^{\circ} \mathrm{I}$, March 1996, p. 43-66. Sur les particularités de la pratique généalogique, entre le monde de la recherche scientifique et les univers de l'érudition familiale : Tiphaine Barthelemy et Marie-Claude Pingaud (dir.), La Généalogie entre science et passion, Paris, Éd. du Comité des travaux historiques et scientifiques, 1997.

17. "Şecer» : "ağaç, dirhat" (Ahmed Vefik, Lehce-i Osmânî, op. cit, p. 824) ; Ferit Develioğlu, Osmanlıca-Türkçe Ansiklopedik Lûgat, Ankara, Aydın Kitabevi, 1999 (ı6 éd.), p. 982 ; Diran Kelekian, Dictionnaire..., op. cit., p. 721. Ahmed Vefik définit ainsi şecere comme şecere-nâme, mais également comme silsile-nâme en forme d'arbre ("ağaç şeklinde kol kol silsile-nâme", Lehce-i Osmânî, op. cit, p. 824). L'équivalent persan (shajarat, shajara) signifie également arbre ou arbre généalogique (Francis Joseph Steingass, A Comprehensive Persian-English Dictionary, Beirut, Librairie du Liban, 1975 (réimp.), p. 735).

I8. Mehmed Zeki Pakalın, Osmanlı Tarih Deyimleri ve Terimleri Sözlüğ̈̈, 3 vol., Istanbul, Millî Eğitim Bak., 1993 (rééd.), vol. III, p. 314. On trouvera une description précise de şecere de tarikat dans Zeki Oral, «Turgut Oğulları, Eserleri - Vakfiyeleri», Vakıflar Dergisi, vol. III, I956, p. 47 ; et une autre de descendants de conquérants dans Heath W. Lowry, Ismail E. Erünsal, "The Evrenos Dynasty of Yenice Vardar : Notes and Documents on Haci Evrenos and the Evrenosogulları : A Newly Discovered Late-I $7^{\text {th }}$ Century Şecere (Genealogical Tree), Seven Inscriptions on Stone and Family Photographs", Osmanlı Araşttrmalar / The Journal of Ottoman Studies, vol. XXXII, 2008, p. I2-I9. Citons également la description suivante : "In I93I I was shown their familial tree. It is set out on a roll about seven feet long and nine inches wide, headed by the doxology and a long exordium, the first five lines in black followed by the gradual introduction of couloured lines until they develop into a regular sequence of black, blue, red, yellow and green in that order. It ends with the above mentioned S. Muhammad, is dated I2IS Rumi (I799-I800), and bears the seal of various orthodox religious authorities" (Cecil J. Edmonds, Kurds, Turks and Arabs, London, Oxford University Press, 1957, p. I88-I89; avec une photographie de l'arbre, déroulé et brandi par deux membres de la famille, p. I46-I47). L'auteur évoque les détenteurs de l'arbre sous le nom d'Ibrahimi, branche ainnée d'une importante chefferie du Kurdistan méridional, les Kakai. Il s'agit probablement d'une famille de Khândân, lecteurs-récitateurs des textes sacrés des $A b l-i$ haqq. Je remercie Catherine Mayeur-Jaouen pour cette précision. 
simple liste de noms, sans disposition rigoureuse, comme si l'auteur, sur un mode calligraphique habituel chez les scribes, remplissait la page sans schéma préalable, au gré de l'espace restant. Il arrivait que quelques dates fussent inscrites, généralement celle du décès; que les noms fussent reliés les uns aux autres par des traits ou des pointillés, ou dans d'autres cas, entourés par des cercles ${ }^{19}$, parfois simples, parfois doubles ou triples, de taille variable selon l'importance prêtée à l'individu par l'auteur du seçere. Le lignage était alors mis en valeur ; ainsi qualifiait-on de şecereli celui dont la bonne naissance était prouvée par l'existence d'un arbre ${ }^{20}$; et aujourd'hui encore, cette notion est associée au prestige social dérivé d'une ascendance illustre ${ }^{21}$.

Une dernière forme - celle de soyağacı ${ }^{22}$ - fut employée plus tardivement, dans la seconde moitié du XIX siècle ; à la fois parce qu'elle renvoyait d'un point de vue sémantique comme formel à la représentation en arbre, mais aussi parce qu'elle intégrait la notion d'ascendance comme race (soy, terme polysémique signifiant également famille, lignage ou descendants), au cœur de l'imaginaire politique turc à l'époque ${ }^{23}$. À telle enseigne qu'elle domina la terminologie généalogique de la Turquie républicaine - à l'origine de nouveaux termes, tels soy kütü̈̆̈̈ (arbre généalogique) ${ }^{24}$ ou soybilim (généalogie) ${ }^{25}$, soybilimci (généalogiste) ${ }^{26}$ - même si le mot şecere continue d'être employé aujourd'hui ${ }^{27}$.

\section{Une pratique discrète}

Les Ottomans parlaient donc généalogie. Ils tardèrent pourtant à constituer des arbres. Les plus anciens dont nous disposons furent produits par des familles intégrées dans l'État en expansion au XIV ${ }^{e}$ siècle : les descendants d'illustres figures de princes ou de conquérants, ainsi Turgut ou Evrenos; les héritiers de célèbres maîtres soufis, tels les Cemali ${ }^{28}$. De leur côté, les sultans Ottomans se contentaient de préserver les mosaïques chrétiennes et les représentations d'arbres de Jesse qu'ils découvraient au gré des villes conquises - on trouve encore de

19. Nous disposons, par exemple, de deux types différents de şecere pour l'illustre famille Cemali : l'un dispose des noms reliés par des pointillés, l'autre des noms en cercles, de taille différente, reliés par des traits (Yusuf Küçükdağ, II. Bayezid, Yavuz ve Kanûnî Devirlerinde Cemâlî Ailesi, Istanbul, Aksaray Vakfi Yayınları, 1995, p. 206-207).

20. Diran Kelekian, Dictionnaire..., op. cit., p. 721.

2I. Yalçın Kocabay, Türkçe-Fransızca Büyük Sözlük, Ankara, Tisamat, 1998, p. 19.

22. Le terme apparaît dans Sir James W. Redhouse, Redhouse Türkçe-İngilizce, op. cit., p. IO28; Ahmet Vefik ne le mentionne pas.

23. Du reste, dans certains dictionnaires de la fin de la période, les termes neseb ou silsile étaient entre autres traduits par "race» (Diran Kelekian, Dictionnaire..., op. cit., p. 689, p. I 273).

24. Ahmet Rıza Yalt, Grand Dictionnaire Français-Turc, Istanbul, Ararat Yay., I971, p. 518 ; Tahsin Saraç, Büyük Fransızca-Türkçe Sözlük, Istanbul, Adam, 1985, p. 94.

25. Tahsin Saraç, Büyük..., op. cit., p. 658.

26. Ibid.

27. Yalçın Kocabay, Türkçe-Fransızca..., op. cit., p. 893 ; «şecereli» : «dont la naissance est prouvée par un arbre généalogique» (ibid., p. 893).

28. Heath Lowry et İsmail Erünsal, «The Evrenos Dynasty...», art. cit. ; Yusuf Küçükdağ, II. Bayezid..., op. cit. 
remarquables généalogies du Christ sur les murs des églises et des monastères, à Istanbul, en Anatolie dans les Balkans ${ }^{29}$. Ils étaient pourtant musulmans, et la généalogie était un genre hautement islamique ${ }^{30}$.

Le Prophète avait entériné son rôle tribal éminent, anciennement cultivé dans la péninsule arabique ${ }^{31}:$ "apprenez assez de généalogie pour connaître vos ashâa et accomplir les devoirs imposés par la parenté ${ }^{32}$. Des les premiers siècles de l'islam, la généalogie s'était constituée en pratique écrite, au contact de la poésie (les versets polémiques, en particulier), des récits tribaux et de la science antéislamique des ansab dont elle se nourrissait, de l'isnâd à laquelle elle apportait un cadre de validité, et de l'historiographie dont elle devenait une discipline auxiliaire. Le développement du culte du Prophète et de ses descendants assurait une base doctrinale au fait même d'étudier les généalogies, et plus généralement à la sainteté héréditaire ${ }^{33}$. Les circonstances politiques et sociales du temps favorisaient l'expansion du genre : les tentatives de tribus affaiblies de se rattacher aux Quraysh, la conscience des conquérants arabes d'une mission historique universelle, le souci de classification chronologique des événements, l'apparition d'une nouvelle aristocratie dans la société musulmane. La généalogie fit son entrée dans les nouveaux systèmes de gouvernement central (dîwân) soucieux d'identifier les nouveaux convertis ${ }^{34}$ : au VIII ${ }^{\mathrm{e}}$ siècle, les Omeyyades commandèrent des registres complets de généalogies. Des le XIII ${ }^{\mathrm{e}}$ siècle, des ashrâf (descendants du Prophète) se déplaçaient munis de leur kitâb al-nasab (nasabnâme, "livre de généalogie»)35. Des mises en forme de toutes sortes (souvent sous forme de squelette ou d'arbre à forme humaine) furent ensuite imaginées par des générations successives de lettrés ${ }^{36}$, entre le $\mathrm{Ix}^{\mathrm{e}}$ siècle, riche en érudits soucieux de restituer l'essence d'ou-

29. Un des cas les plus admirables et les mieux documentés est la généalogie de l'Église Chora. Les plus hautes figures de la Bible y entourent le médaillon du Christ représenté en Pantecrator dans le dôme sud, et celui de la Vierge à l'Enfant dans le dôme nord. On en trouvera une présentation détaillée dans Paul A. Underwood, The Kariye Djami, 4 vol., 1966-1975, vol. I, p. 49-59, vol. 2, p. 46-85.

30. Franz Rosenthal, «Nasab», Encyclopédie de l'Islam (dorénavant EI²), vol. VII, Leiden, E. J. Brill, 1993, p. 968 ; Franz Rosenthal, A History of Muslim Historiography, Leiden, E. J. Brill, I968 ( $2^{\text {nd }}$ ed.), p. 95-IOO ; Eduard de Zambaur, Manuel de généalogie et de chronologie pour l'histoire de l'Islam, Hanovre, Librairie Orientaliste Heinz Lafaire, 1927 (pour une comparaison notamment avec les dynasties seldjoukides).

3I. Édouard Farès, L'honneur chez les Arabes avant l'Islam, Paris, Adrien-Maisonneuve, I932, p. 8I-88. On trouvera une étude complète sur les généalogies tribales dans Werner Caskel, Gamharat an-Nasab. Das Genealogische Werk des Hišàm ibn Muhammad al-Kalbî, Leiden, E. J. Brill, 1966, 2 vol. ; on la complétera par une lumineuse analyse proposée par Meir Jacob Kister et Martin Plessner, «Notes on Caskel's Ğamharat an-Nasab», Oriens, vol. XXV-XXVI, 1976, p. 48-68.

32. Charles Pellat, "Hasab wa-Nasab», $\mathrm{EI}^{2}$, vol. III, I965, p. 245-246.

33. Catherine Mayeur-Jaouen, "Le saint musulman...", art. cit.

34. 'Abd al-'Azîz al Dûrî, The Rise of Historical Writing Among the Arabs, (ed. and translated by Lawrence I. Conrad), Princeton, Princeton University Press, I983, p. 50-51, 75, I36-138, I46.

35. C'est ce que révèle l'étude de l'hagiographie des saints (Catherine Mayeur-Jaouen, «Le saint musulman en père de famille», art. cit.).

36. Oscar Löfgren, «Dagfal und Di'Bil als Gewährsmänner der Südarabischen Sage», dans Studi Orientalistici in onore di Giorgio Levi Della Vida, Rome, Istituto per l'Oriente, 1956, 2 vol., vol. II, p. 94-IOI. 
vrages généalogiques par le recours à la métaphore de l'arbre, et le XIv siècle, quand un Ibn Khaldun recommandait (à qui voudrait mettre en forme des relations de parenté) l'usage de l'arbre avec une colonne ('amûd) centrale, selon une structure segmentaire - l'expression 'asabiyya qu'il employait renvoie précisément aux nerfs ${ }^{37}$. À l'époque moderne et contemporaine, les principales dynasties du monde arabe tinrent à représenter leur lignée sous des formes sans cesse plus élaborées, avec dans certains cas, l'appui d'érudits européen $s^{38}$. Aujourd'hui encore, quantité de posters sont vendus au Caire ou dans des capitales voisines qui représentent les Abl al-bayt ("Gens de la Maison», ou membres de la famille du Prophète) $)^{39}$.

Les sultans ottomans avaient eux-mêmes bien des raisons de s'approprier une culture généalogique développée chez leurs sujets : l'attachement à la transmission évoqué plus haut ; un encouragement constant à l'insertion des études généalogiques (ilm al-ensab ou, sous une forme persane, ilm-i neseb, ilm-i ensa $b^{40}$ ) dans l'étude des sciences classiques ; une garantie institutionnelle offerte aux conditions de leur enseignement (icazetnâme, "certificat d'aptitude», dérivé du principe de licence, 'idjaza) ; des relations de proximité avec les milieux confrériques et leurs chefs ; une reconnaissance de la noblesse acquise ou héritée ${ }^{41}$. Le fait est, cependant, que les généalogistes étaient et restèrent des figures discrètes de leur littérature et de leur historiographie. Était-ce parce que dans la péninsule arabe préislamique, la généalogie était une «expression primaire de la conscience historique» (Franz Rosenthal) ${ }^{42}$, alors que, dans le monde turc dont les Ottomans étaient issus, elle en était une manifestation secondaire ? Était-ce parce que les conflits entre les chefferies semi-nomades anatoliennes se posaient en des termes différents de ceux qui opposaient les grandes familles arabes détribalisées ? Étaitce parce que les sultans, en quête de légitimité auprès du califat sunnite, n'avaient pas dans les premiers temps de leur règne une conception de leur rôle historique aussi ambitieuse que les premiers conquérants de l'islam ? Il faudrait consulter les historiens médiévistes à ce sujet. Tâchons pour notre part de reconstituer les grandes lignes de l'émergence du genre.

37. Franz Rosenthal, «Nasab», art. cit. ; Zoltan Szombathy, «Genealogy in Medieval Muslim Societies", Studia Islamica, vol. 95, 2002, p. 5-35. Je remercie Catherine Mayeur-Jaouen pour ses éclaircissements.

38. On trouvera un arbre végétal de la dynastie koweitienne constitué par un membre de la famille dans Alan de Lacy Rush, Al-Sabah. History and Genealogy of Kuwait's Ruling Family, London, Ithaca, 1987. On trouvera des représentations sophistiquées des dynasties actuelles dans Alan de Lacy Rush, Ruling Families of Arabia. Family Trees, Melksham, Oxon, Archive Editions, I99I.

39. On en trouvera un bel exemple, reproduit et commenté, dans Catherine Mayeur-Jaouen, AlSayyid Ahmad al-Badawî. Un grand saint de l'islam égyptien, Le Caire, IFAO, 1994, p. I43-I45. La rédaction de ce paragraphe doit beaucoup aux échanges avec l'auteure.

40. "Genealogy ", Redhouse Türkçe-İngilizce Sözlük, Istanbul, Sev, 1997 (rééd.), p. 529 ; Diran Kélékian, Dictionnaire..., op. cit., p. 157.

4I. Charles Pellat, «Hasab wa-Nasab», art. cit.

42. Franz Rosenthal, «Nasab», art. cit., p. 968. 


\section{Du côté de chez Osman : naissance et développement d'un genre}

Conquête après conquête, la réputation grandissante des Ottomans commençait de susciter l'intérêt des généalogistes européens : la plus ancienne représentation de la maison d'Osman qui nous est connue date de la fin du $\mathrm{Xv}^{\mathrm{e}}$ siècle. Elle fut réalisée sous le règne de Bayezid II (I48I-I5I2) par Felix Petancius. À la fin $\mathrm{du} \mathrm{XVI}^{\mathrm{e}}$ siècle, les sultans commandèrent leurs premières généalogies ${ }^{43}$. À partir de cette période, les silsilenâme se multiplièrent ${ }^{44}$. Pourquoi alors ? Parce que la culture dynastique ottomane disposait désormais de trois appuis, aussi solides à la fin du XVI ${ }^{e}$ siècle qu'ils avaient été absents ou en gestation au début de ce même siècle : depuis les premiers récits d'Ibn Kemal, Neşri et Aşıkpaşazâde sous le règne de Bayezid II, les histoires de la dynastie ottomane s'étaient multipliées - Robert Mantran en recense vingt et une pour le seul règne de Soliman le Magnifique (I520-1566) ${ }^{45}$; le genre généalogique, moins développé, était néanmoins identifiable dans les travaux de Yusuf b. Abdullatif ou de Mehmed b. Ramazân ${ }^{46}$. Les Ottomans possédaient des reproductions d'arbres, européens - on en trouvera au musée de Topkap 1-, mais également timurides et mongols dont il est établi qu'ils $s^{\prime}$ inspirèrent ${ }^{47}$. Surtout, depuis la conquête des provinces arabes en 1516-1517, la dynastie ottomane étaient engagée dans une entreprise de légitimation sunnite au sein de laquelle le genre généalogique trouvait sa place.

Entre-temps, l'arbre en tant que forme métaphorique avait fait son apparition, sous la plume d'artistes vénitiens, au milieu du $\mathrm{XVI}^{\mathrm{e}}$ siècle $^{48}$. Sur un arbre anonyme daté de 1603 , on distinguait clairement les sultans, mais également certains de leurs fils ${ }^{49}$. Selon Gül İrepoğlu, il fallut cependant attendre le règne d'Abdülhamid Ir (1774-I789) pour que l'arbre soit introduit à la Cour, sous l'effet d'une inspiration européenne, mais à une époque où l'identité chrétienne qui lui était originellement consubstantielle était moins marquéeso. En effet, sur un exemplaire conservé au musée de Topkapı, l'influence de la peinture européenne du XVIII ${ }^{e}$ siècle (telle qu'elle fut adoptée par l'école du peintre arménien Rafael,

43. Julian Raby, «From Europe to Istanbul», dans Filiz Çağman et al., The Sultan's Portrait. Picturing the House of Osman, Istanbul, İşbank, 200o, p. 137.

44. On en trouvera de magnifiques exemples dans Filiz Çağman et al., The Sultan's Portrait..., op. cit., p. $28 \mathrm{I}-284$.

45. Robert Mantran, "L'historiographie ottomane à l'époque de Soliman le Magnifique», dans Gilles Veinstein (dir.), Soliman le Magnifique et son temps, Paris, La Documentation française, I992, p. 29.

46. Serpil Bağc1, «From Adam to Mehmed III : Silsilenâme», dans Filiz Çağman et al., The Sultan's Portrait..., op. cit., p. I88.

47. Gülrü Necipoğlu, "The Serial portraits of Ottoman Sultans in Comparative Perspective», dans Filiz Çağman et al., The Sultan's Portrait..., op. cit., p. 22-6I.

48. Voir par exemple une gravure de l'arbre de la famille ottomane dans Francesco Sansovino, Sommario et alboro delli principi Othomani, Venise, 1567 (reproduit dans Filiz Çağman et al., The Sultan's Portrait..., op. cit., p. 236).

49. Daté de I603; Ertugrul est au pied, Ahmed III au sommet ; les termes sont en latin ; l'œuvre est conservée à Greven, Sammlung Hans Galen (Filiz Çağman et al., The Sultan's Portrait..., op. cit., p. 3I4).

50. Gül İrepoğlu, «Innovation and Change», dans Filiz Çağman et al., The Sultan’s Portrait..., op. cit., p. 378-439. 
mort en 1780 , possible auteur de l'arbre) est très nette, qu'il s'agisse de la forme choisie (peinture à l'huile sur grand format) ou de la représentation du paysage à l'arrière plan. Cela dit, les Ottomans faisaient usage d'un genre de représentation dont ils avaient désormais la maîtrise : ce furent les miniatures de Levnî exécutées sous le règne d'Ahmed III (I703-I730) qui furent copiées à la fin du XVIII ${ }^{\mathrm{e}}$ siècle pour faire figurer les médaillons des sultans répartis avec équilibre sur toute la hauteur de l'arbre; on les reconnaît bien, reliés les uns aux autres (du tronc où figure le souverain régnant, au sommet où apparaît le fondateur Osman) par un large ruban, métaphore du lignage ${ }^{s^{2}}$. L'ordre de succession n'est pas toujours restitué aussi fidèlement : sur une représentation composée sous Selim III (I789-I807), on identifie tout aussi clairement le tronc, à partir duquel un ensemble de feuilles se déploie vers le ciel $^{13}$, mais la longue guirlande végétale qui relie les sultans ne permet de reconstituer ni leurs liens de parenté, ni leur ordre de succession ${ }^{54}$.

Une interprétation différente de l'apparition (légèrement ultérieure) de l'arbre, d'origine à la fois ottomane et occidentale, a été récemment proposée par Günsel Renda. Dans son célèbre Tableau, Mouradgea d'Ohsson (I740-I807) raconte qu'il avait obtenu de faire copier les portraits de l'album des sultans conservé à Topkapı. Lors d'un séjour à Paris, il avait présenté ces copies au roi de Suède, Gustav III, lequel très intéressé, lui avait commandé la composition d'arbres généalogiques à partir de ces portraits - c'est la raison pour laquelle le musée du Gripsholm Palace possède aujourd'hui une représentation d'arbre généalogique des sultans. Des trois tableaux commandés, d'Ohsson en rapporta un à Istanbul en I792. Il le montra au sultan. Celui-ci, étonné par la ressemblance avec les portraits de Topkapı sur laquelle son interlocuteur l'éclaira, en ordonna des copies, qui produisirent un effet sensationnel sur les dignitaires de la Cour, avant d'être reproduites en Europe $^{\text {s. }}$. Si j'insiste sur cette version des faits, c'est qu'elle éclaire très largement les modes d'influence mutuelle, les transferts culturels qui se développaient alors entre Istanbul et les capitales occidentales, autant qu'elle souligne l'intérêt qu'accordaient les Ottomans à la politique des symboles : de même qu'au Xvi ${ }^{\mathrm{e}}$ siècle la généalogie s'était développée dans le cadre d'un effort de légitimation dynastique, de même l'arbre de la fin du XVIII ${ }^{\mathrm{e}}$ siècle fut conçu comme vitrine du régime.

Il le fut davantage encore quelques années plus tard, lorsque de nouveaux supports furent mis à la disposition des artistes : on trouve des gravures imprimées et

5I. Ibid., p. 387 ; avec des miniatures (ibid., p. 406-4IO). Voir aussi Günsel Renda, "Osmanlı Sultanlarının Soyağacı", P : [Portaka] Sanat Kultür Antika Dergisi, n² 2, été 1996, p. 84.

52. Gül İrepoğlu, «Innovation...», art. cit., p. 388.

53. Topkapı Sarayı Müzesi, I7/I33 (cité par Günsel Renda, «Portraits : The Last Century», dans Filiz Çağman et al., The Sultan's Portrait..., op. cit., p. 516).

54. C'est également le cas d'un arbre anonyme daté de I866-I867 (Günsel Renda, «Portraits...», art. cit., p. 518).

55. Günsel Renda, "Illustrating the Tableau Général de l'Empire Othoman», dans The Torch of the Empire. Ignatius Mouradgea d'Ohsson and the Tableau Général of the Ottoman Empire in the Eighteenth Century, Istanbul, Yapı Kredi Yayınları, 2002, p. 66-67. On comparera cette nouvelle version à celle que donnait Günsel Renda avant de prendre en compte un document manuscrit d'Ohsson trouvé à la Lund University Library (Günsel Renda, «Portraits... », art. cit., p. 442-446 ; Günsel Renda, «Osmanlı Sultanlarının Soyağacı», art. cit., p. 87). 
coloriées dans les premières décennies $\mathrm{du} \mathrm{XIX}^{\mathrm{e}}$ siècle ${ }^{56}$, des reproductions de qualité variable dans des revues populaires du $\mathrm{XX}^{\mathrm{e}}$ siècle ${ }^{57}$. Dans l'une d'elles, datée de l'époque de Mehmed V (I909-I9I8), les éléments végétaux sont plus disparates; le motif central ne ressemble que de loin à un arbre. Les sultans sont agglutinés autour du souverain régnant, dont le portrait au centre est distingué par la double rangée de ses ancêtres qui l'entourent et la reproduction de son monogrammes ${ }^{88}$.

\section{Les arbres à sultan}

Analysés sur l'ensemble de la période, ces arbres présentent deux constantes. Contrairement à bien des arbres royaux ou princiers en usage en Europe occidentale, il semble que la représentation généalogique n'était pas conçue comme exhaustive ; ni les branches cadettes, ni les familles princières alliées n'étaient signalées; les Ottomans n'estimaient pas utile d'adopter des formes plus complètes qui leur étaient consacrées sur des gravures européennes. L'expression «arbre de famille» employée par certains collègues parait inappropriée ${ }^{59}$ - Pakalın considère qu'un arbre (şecere) «montre l'ensemble des branches et des enfants» (bütün kolları ve avlarların gösteren $)^{60}$, ce qui était loin d'être toujours le cas.

Seconde constante, ces arbres proposaient moins une représentation généalogique qu'un tableau dynastique. Encore, celui-ci était-il souvent sommaire : les noms des sultans n'étaient pas toujours indiqués, et l'ordre de succession semblait moins importer que le nombre de sultans. Ici comme en bien des domaines, les Ottomans portaient bien leur nom : il leur suffisait de se signaler comme descendants d'Osman ; il leur importait de souligner le caractère extraordinaire de leur continuité, non seulement au regard des longévités dynastiques moyennes ${ }^{61}$, mais de l'apparente tranquillité avec laquelle ils avaient traversé l'histoire.

Il est vrai que la dynastie ne fut que rarement menacée - entre autres raisons par la possible absence d'héritiers mâles -, et qu'il ne fut jamais nécessaire d'aller chercher au-delà du frère, neveu ou cousin germain pour assurer la succession ${ }^{62}$.

56. Filiz Çağman et al., The Sultan's Portrait..., op. cit., p. 285. La première gravure d'un portrait de sultan publiée à l'étranger, à Londres, date de 1803 (Bahattin Öztuncay, The Photographers of Constantinople. Pioneers, Studios and Artists from Nineteenth century Istanbul, Istanbul, Aygaz, 2006, $2^{\mathrm{e}}$ éd., 2 vol., vol. I, p. 37).

57. Günsel Renda, «Portraits...», art. cit., p. 444-446.

58. Stéphane Yerasimos et al., Istanbul au temps des derniers Ottomans, Paris, éd. du Chêne, 2003.

59. Günsel Renda, "Portraits...», art. cit., p. 516. Il est curieux de constater que les auteurs de cet ouvrage notent les influences européennes, mais ne signalent pas en quoi elles sont limitées par l'absence de prise en compte des autres membres de la famille.

6o. Mehmed Zeki Pakalın, Osmanlı Tarih Deyimleri ve Terimleri Sözlü̈̆ü, Istanbul, Millî Eğitim Bak. Yay., 1993 (rééd.), 3 vol., vol. III, p. 314.

6I. Voir John E. Morby, Dynasties of the World: A Chronological and Genealogical Handbook, Oxford / New York, Oxford University Press, 1989.

62. Anthony D. Alderson, The Structure of the Ottoman Dynasty, Oxford, Clarendon, 1956, p. 4 et I4. La plus grave menace survint en I640, lorsque Murad IV ordonna, sur son lit de mort, l'exécution de son frère Ibrahim, le seul membre mâle de la famille, sauvé in extremis par un stratagème de leur mère. Sur les "périls de la succession », voir Nicolas Vatin et Gilles Veinstein, Le Sérail ébranlé. Essai sur les morts, dépositions et avènements des sultans ottomans XIV -XIXe siècle, Paris, Fayard, p. I09-182. 
On aurait pourtant tort de croire que cette continuité fut naturelle, qu'elle ne fut pas conditionnée. Tout fut entrepris pour que la dynastie ne perdît jamais le monopole de l'État : les alliances princières furent abandonnées dans les premiers siècles ; la pratique du fratricide fut appliquée systématiquement par plusieurs sultans ; le confinement au Palais devint une règle observée après Mehmed III (1595-1603), ce qui réduisit à presque rien le statut politique des princes, qui n'étaient pas autorisés à avoir d'enfant - il fallut attendre I86I pour qu'un père accédât au trône $e^{63}$.

Le principe d'unité du pouvoir gouvernait l'ordre des symboles : la vitrine généalogique du régime, c'était le sultan, «l'unique soleil du sultanat»", représenté partout à partir de Mahmud II (I808-I839), dans les bâtiments publics, dans les casernes et les écoles. Avec les princes et les sultanes, l'opposition était nette : de même qu'ils ne pouvaient prétendre au partage familial du pouvoir, de même n'avaient-ils droit à aucune visibilité. Nul ne considéra jamais qu'ils constituaient une famille royale (au sens où on entend cette expression en Europe occidentale), dont les membres auraient été dignes de figurer auprès du souverain - on pense par exemples aux photographies de la Reine Victoria entourée de sa famille ${ }^{65}$. Ahmed III fut l'un des rares sultans qui estima nécessaire de se représenter aux côtés de ses fils ${ }^{66}$. Depuis la toute fin du Xvi ${ }^{\mathrm{e}}$ siècle, les princes étaient des «inconnus» (pour reprendre l'expression de François Georgeon) ${ }^{67}$. Autant les chroniqueurs vantaient les mérites des sultans et de leurs plus insignes serviteurs, autant ils estimaient superflu de devoir signaler des cousins peu susceptibles d'être sollicités en cas d'une très improbable rupture dynastique ; certains poussèrent même la négligence jusqu'à ne pas inscrire le nombre et le nom de certains şehzâde (fils de sultan) ${ }^{68}$. Il faut bien reconnaître que le sujet était délicat : lors de leur avènement au trône, les sultans Murad III en I574 et Mehmed III en I595 avaient massivement pratiqué le fratricide ${ }^{69}$.

Fait nouveau cependant, sous les Tanzimat et surtout après le voyage européen du sultan Abdülaziz en 1867, les princes furent intégrés à la mise en représentation du pouvoir ; ils ne boudaient pas leur plaisir d'inclure leur meilleur portrait dans leur carte de visite $^{70}$; on les trouvait représentés ensemble, sur quelques

63. Anthony Alderson, The Structure..., op. cit., p. 35 .

64. Formule employée par un chroniqueur de l'époque de Selim II citée par Nicolas Vatin et Gilles Veinstein, Le Sérail ébranlé..., op. cit., p. 83. Sur les liens entre l'unité du pouvoir, le statut des membres de la famille, et les pratiques de succession : $i b i d .$, p. 82-85.

65. Voir par exemple la photographie de la famille royale prise à Osborne en I857 (Monica Charlot, Victoria. Le pouvoir partagé, Paris, Flammarion, 1989, p. 248-249). Nuançons aussitôt l'opposition : bien des généalogies royales occidentales avaient moins le souci de dire des liens de parenté que de rendre compte d'un ordre de succession (Bernard Guénée, «Les généalogies entre l'histoire et la politique. La fierté d'être Capétien, en France, au Moyen Âge ", Annales ESC, vol. XXXIII, n ${ }^{\circ}$, I978, p. I-I4).

66. Je remercie Gilles Veinstein pour cette indication.

67. François Georgeon, Abdülhamid II. Le sultan calife, Paris, Fayard, 2003, p. 53.

68. Leslie Peirce, The Imperial Harem. Women and Sovereignty in the Ottoman Empire, Oxford, Oxford University Press, 1993, p. IO4.

69. Nicolas Vatin et Gilles Veinstein, Le Sérail ébranlé..., op. cit., p. I70-I7I.

70. Voir les cartes de visite des şehzade Kemaleddin, Mehmed Reşad, Mahmud Celaleddin, Abdulhamid, 
photographies $^{71}$. Mais ces nouveautés ne tombaient sous l'œil que de quelques élites ; et si les damad, les gendres du sultan, étaient cités en bonne place et sous leur meilleure mise dans les revues officielles, ils l'étaient autant, voire davantage, en tant que dignitaires du régime (souvent membres du Conseil d'État à l'époque hamidienne) qu’apparentés à la famille impériale. Les Ottomans ignoraient à ce point l'identité des princes que, sur des photographies de l'époque, certains furent confondus avec des fils de dignitaires ${ }^{72}$.

La famille impériale n'était certes pas totalement désintéressée par le recensement des siens. Pour preuve, il existe un registre de la maison d'Osman, un hanedan defteri, interrompu à la fin de l'Empire, avec comme dernier membre indiqué Neslişah Sultan, née en $192 \mathrm{I}^{73}$. Aux archives de la Présidence du Conseil, on trouve quantité de documents sur les princes et les princesses, sur les dépenses occasionnées lors des mariages. Mais il faut y voir, me semble-t-il, une pratique traditionnelle de l'administration ottomane, ce goût particulier pour le recensement des ressources, davantage que la valorisation idéologique d'un groupe lié au sultan. Jamais personne n'estima nécessaire d'écrire une histoire détaillée de la maison d'Osman - comme des Habsbourg ou des Romanov par exemple. Il fallut attendre la toute fin de l'Empire pour que ses statuts en soient définis sur le papier ${ }^{74}$. Ils obéissaient à un principe simple : la maison impériale ne devait concerner qu'un nombre limité d'individus. Aussi différenciait-on l'appartenance à la famille (entendue comme aile, avec ses descendants, parents et alliés) à l'appartenance à la maison (au sens de hanedan), bien plus restrictive. Au sein de la maison, deux catégories étaient distinguées : d'une part, les $a z a$, princes du sang, qui regroupaient le sultan, les şehzâde (descendants patrilinéaires du sultan), les sultan (filles des deux précédentes catégories); d'autre part, les mensub (apparentés) qui désignaient premièrement les époux et épouses des $a z a$, deuxièmement les enfants des sultan; les descendants des premiers perdaient leur titre dynastique à la seconde génération ; ceux des seconds, dès la première ${ }^{75}$. Alors qu'au gré des naissances, l'arbre généalogique de la famille s'étendait, celui de la maison impériale gagnait ou perdait de nouvelles branches. Bref, l'idéologie a-nobiliaire ottomane avait été assouplie mais préservée : tout avait été fait pour que le soleil

Yusuf İzzedin, datées des environs de 1870 (Engin Özendes, Abdullah Frères. Osmanlı Sarayının Fotograf̧̧ılar, Istanbul, Yapı Kredi Yayınları, 1998, p. 19, 51, 147, 174, 225). Voir également la photographie du prince Abdülhamid (le futur Abdülhamid II) : Yaşamları ve Yapıtlarıyla Osmanlilar Ansiklopedisi, Istanbul, Yapı Kredi Yay., I999, 2 vol., vol. I, p. 52.

7I. On trouvera des photographies (individuelles, avec quelques fratries) de şehzâde et de sultan dans Bahattin Öztuncay, The Photographers..., op. cit., vol. 2, p. 352-372.

72. Sur la couverture de Halil Halid, The Diary of a Turk, London, Adam and Charles Black, 1903, une photographie de fils de dignitaires est présentée à tort comme celle de princes.

73. Hanedan-ı Âl-i Osman Defteri cité in İlber Ortaylı, Osmanlı Toplumunda Aile, Istanbul, Pan, 2000, p. 36.

74. On trouvera le dernier règlement du hanedan, daté de janvier 1920, dans Osman Selaheddin Osmanoğlu (dir.), Ali Vâsıb Efendi. Bir Şehzadenin Hâtırâtı. Vatan ve Menfâda Gördüklerim ve İsittiklerim, Istanbul, Yapı Kredi Yay., 2004, p. 409-410.

75. Voir Osman Selaheddin Osmanoğlu, Ali Vâsıb Efendi..., op. cit., p. 4IO ; Kerime Senyücel, Hanedan'in Sürgün Öyküsü, Istanbul, Timaş, 2009, p. I3. Je remercie chaleureusement Edhem Edhem pour ses précisions. 
du sultanat ne rayonnât jamais, hors de la figure du sultan et au-delà de son règne, que de quelques rayons.

L'examen historiographique permet de comprendre les circonstances historiques et politiques qui firent que les sultans ottomans tardèrent à entrer en généalogie. Il aide à éclairer les raisons pour lesquelles ils s’approprièrent la pratique, et à identifier les sources d'inspiration qui furent les leurs. Il invite également à considérer que les généalogies, comme les chroniques et les portraits, reflétaient autant les spécificités de la maison d'Osman qu'elles traduisaient des références visibles dans d'autres systèmes impériaux. On en prendra la mesure en reliant la pratique généalogique aux conceptions de la noblesse et aux systèmes de représentation de soi, tels qu'ils avaient cours chez les hauts dignitaires ottomans ${ }^{7}$.

\section{Grandes familles sans généalogie}

\section{Des serviteurs du sultan et de leur noblesse}

On trouve bien des raisons de comparer les dignitaires au Grand Seigneur. Ghazis et pachas avaient combattu à ses côtés ; ils servaient sous le signe de son monogramme (tughra); ils vivaient à son image, dans de vastes maisons dotées d'un harem, entourées d'une domesticité pléthorique ; ils avaient des esclaves, ne sortaient qu'accompagnés d'une suite imposante et bigarrée. Au XIX ${ }^{e}$ siècle, la frontière entre les «sultans bourgeois ${ }^{77}$ et les désormais "fonctionnaires» était plus ténue encore : les uns et les autres avaient adopté les mêmes nouveautés techniques (l'éclairage au gaz, les calèches dernier cri) et culturelles (la lecture du roman, les manières de table), tout en continuant d'adhérer pleinement aux usages de la Cour, entre amour des belles lettres ( $a d a b)$ et observance religieuse ; nombreux étaient ceux qui continuaient de pratiquer la polygamie, alors qu’à Istanbul le modèle de la famille nucléaire dominait ${ }^{78}$. Comme le sultan, les dignitaires gouvernaient en autocrate, à la tête de leur ministère ou de leur province. Comme lui, ils ne payaient pas l'impôt. Comme lui, ils étaient riches et voulaient l'être davantage. Ils s'identifiaient à la maison d'Osman - on les disait Osmanl.

Il ne faudrait pas pour autant oublier qu'une barrière fondamentale, réelle et symbolique, séparait les dignitaires de leur souverain ${ }^{79}$ : ils savaient qu'ils ne pouvaient le copier en tout ; quils ne pouvaient prétendre au partage du pouvoir. Le grand vizir lui-même n'était jamais que vekil, représentant du sultan ; il ne pouvait se revendiquer du service de l'État en dehors de cette référence. Sous Soliman, le

76. On rapportera nos réflexions à l'étude des généalogies d'oulémas proposée par Hans Georg Majer, Vorstudien..., op. cit.

77. François Georgeon, Abdülhamid II..., op. cit., p. I36-I42.

78. Alan Duben, "Turkish Families and Households in Historical Perspective», Journal of Family History, vol. I0, 1985, p. 75-97 ; Alan Duben, "Understanding Muslim Households in the Late Ottoman Period", Journal of Family History, vol. 15, 1990, p. 7I-86; Cem Behar et Alan Duben, Istanbul Households..., op. cit., p. I56-I57.

79. Metin Kunt, «Kulların Kulları», Boğaziçi Üniversitesi Dergisi, Hümaniter Bilimler, vol. III, 1975, p. 29-30. 
célèbre Ibrahim Pacha s'était arrogé le titre (elkab) de kaymakam-i saltanat (lieutenant du sultanat). Le geste était parfaitement inappropriée ${ }^{80}$. Ce Fouquet ottoman le paya de sa vie - même s'il avait déplu pour d'autres raisons. Quo non ascendet ? $^{81}$ Personne ne pensait en ces termes, à l'exception de quelques téméraires qui accrurent leur puissance, protégés par l'éloignement de la capitale et l'affaiblissement du pouvoir central ; pour un temps, tel Tepedenli Ali qui tomba sous le sabre ; pour longtemps, tel le gouverneur général d'Égypte, Muhammed Ali, qui mourut de sa belle mort, et laissa derrière lui une dynastie héréditaire. Il est vrai que les choses avaient changé sous les Tanzimat ${ }^{82}$ : les employés de l'État (memur) avaient des statuts qui les protégeaient de l'arbitraire sultanien. Mais la carrière oscillait encore entre grâce et disgrâce, honneur et relégation, à telle enseigne que les dignitaires se voyaient encore comme des serviteurs de leur maître.

Entre l'habitus de l'identification mimétique au souverain et l'éthique différenciatrice du serviteur, il semble que le rapport des dignitaires à la généalogie s'inscrivait davantage du côté du second terme. Une raison en est qu'il leur était interdit de se référer à ce qui, dans d'autres aires culturelles, fut le terreau qui assura la prospérité de la généalogie, à savoir l'idéologie nobiliaire ${ }^{83}$. Il est certain que les familles anciennes et prestigieuses ne manquaient pas dans l'Empire ottoman ; que certaines d'entre elles avaient le souci de consigner les dates de naissance, de mariage et de décès dans les évangéliaires et les papiers de famille; qu'elles publièrent des généalogies très fournies, dont certaines n’avaient rien à envier aux livres d'or occidentaux ${ }^{84}$. Mais comment ces familles chrétiennes auraientelles pu faire des émules chez des dignitaires musulmans, pour la très grande majorité d'entre eux ? Chez des serviteurs de la Porte qui, s'ils recevaient un sceau individuel par lequel ils cachetaient leurs ordres, ne disposaient (hors exception) d'aucun droit de mettre en avant, qui tel symbole de noblesse, qui telle devise familiale préexistante ? L'idéologie dynastique répondait à un impératif considéré à ce point fondamental par le régime qu'aucun grand, fût-il d'une extraction plus ancienne, n'était en mesure de convertir le prestige de sa noblesse en pouvoir aristocratique ${ }^{85}$.

80. Mehmet Tayyib Gökbilgin, «Ibrâhîm Pasha», EI², vol. III, I968, p. IO23-IO24.

8I. "Jusqu'où ne montera-t-il pas ?». Devise de Nicolas Fouquet (Paul Morand, Fouquet ou le Soleil offusqué, Paris, Gallimard, 1985 (rééd.)). Sur l'ascension et la chute du surintendant des Finances de Louis XIV, voir également Daniel Dessert, Fouquet, Paris, Fayard, 1987.

82. "Réorganisations". On appelle ainsi la période des réformes entreprises entre I839 et I878.

83. On sait qu'en Europe occidentale la généalogie fut une pratique nobiliaire avant d'être adoptée par l'État monarchique (Christiane Klapisch-Zuber, L'Ombre..., op. cit., p. 7, I54, 209).

84. Pour ce qui est des familles phanariotes, voir Eugène R. Rhangabé, Livre d'or de la noblesse phanariote par un Phanariote, Athènes, J. Vastos, I892 (qu'on complétera à la lecture de Mihail Dimitri Sturdza, Grandes familles de Grèce, d'Albanie et de Constantinople. Dictionnaire historique et généalogique, Paris, M. D. Sturdza, 1983). Pour ce qui est des familles chiotes, voir Philip P. Argenti, Libro d'Oro de la noblesse de Chio, London, Oxford University Press, I955, vol. 2. Pour un exemple de famille princière de Valachie, voir Amédée de Foras, Notice historique et généalogique sur les Princes Bessaraba de Brancovan : antiques descendants des anciens voyvodes souverains de la Valachie, etc., Genève, Jules-Guillaume Fick, I889 (avec tableau généalogique, p. 34).

85. On trouvera une réflexion sur les origines comparées de grandes familles de conquérants (Evrenosoğulları, Mihaloğulları, Malkanoğulları, Turhanoğulları) dans Heath W. Lowry, The 
Non seulement la dynastie se considérait comme prééminente, mais elle se voulait autosuffisante : les chroniqueurs se gardaient bien de rappeler l'importance des alliances contractées dans les périodes précédentes avec d'autres familles princières anatoliennes ou balkaniques. À cette pratique abandonnée à partir de la seconde moitié $\mathrm{du} \mathrm{Xv}^{\mathrm{e}}$ siècle, il ne fut jamais question de revenir : la lignée d'Osman était désormais établie, puissante et prestigieuse. Lorsque par exemple, en I866, Abdülaziz envisagea de prendre pour épouse la fille du khédive Ismail, son grand vizir Fuad Pacha l'en dissuada, au motif qu'il ne fallait pas accorder à l'Égyptien trop de poids à la Cour ${ }^{86}$. La dynastie se prolongeait donc à l'aide d'élites qu'elle avait elle-même produites, et qui n'avaient de statut que par elle : les kul (serviteurs de la Porte) auxquels elle retirait toute possibilité de se constituer en lignage, "curbing the potential centrifugal power of the slave elite» ${ }^{87}$; et non les grandes familles d'oulémas dont le prestige lignager (remarquablement accru au XVIII ${ }^{e}$ siècle) aurait trop gagné, aux yeux du souverain, à être associé à la dynastie régnante. Les $k u l$ devaient eux-mêmes se séparer de leurs autres épouses, lors de leur intégration à la famille impériale ${ }^{88}$ : ils perdaient ainsi toute possibilité de se constituer en maison autonome. On comprendra aisément que, dans un système aussi cadenassé, aucune place n’ait été laissée à la pratique généalogique, à ses inventions, à ses discours sur les origines, à un travail d'écriture de la mémoire, un discours sur le rattachement à de prestigieux mythes ${ }^{89}$.

\section{De la représentation de soi}

Dans la continuité de cette explication a-nobiliaire, il en est une autre qu'on voudrait lier au portrait de soi, déjà évoqué pour ce qui est de l'autobiographie par l'État ${ }^{\circ \circ}$. Les dignitaires étaient certes représentés par le pouvoir : ils concouraient autant à la gloire de l'Ottoman qu'à la manifestation de sa souveraineté. Dans la plus célèbre des histoires illustrées (le Şahname Al-i Osman composé par Arifi au milieu du xvi siècle), on voit quantité de vizirs et d'oulémas, tantôt insérés collectivement dans une geste dont les artistes avaient le goût (le siège de Belgrade en 1456 ou de Buda en I54I), ou dans un cérémonial codifié (l'intronisation, beyat), tantôt figurer individuellement au cœur de la miniature en tant que dignitaires honorés par le sultan (Barbaros Hayreddin reçu dans l'intimité du

Nature of the Early Ottoman State, Albany, SUNY Press, 2003, p. 55-78.

86. Anthony Alderson, The Structure..., op. cit., p. 89.

87. Leslie Peirce, The Imperial Harem..., op. cit., p. 7I.

88. Ibid., p. 69-70.

89. Pour une étude sur les généalogies fictives des familles princières tatares dans l'Empire russe, voir István Vásáry, "Russian and Tatar Genealogical Sources on the Origins of the Iusupov Family ", Harvard Ukrainian Studies, vol. 19, 1995, p. 732-746. Pour une comparaison avec des pratiques occidentales au XVIII ${ }^{\mathrm{e}}$ siècle, voir Valérie Pietri, "Les origines de la noblesse de la sénéchaussée de Grasse au XVIII ${ }^{\mathrm{e}}$ siècle», Recherches régionales, $\mathrm{n}^{\circ}$ 185, janvier-mars 2007, p. 32-44. Pour des formes antérieures, voir Christiane Klapisch-Zuber, L'Ombre..., op. cit. ; Roberto Bizzocchi, «La culture généalogique dans l'Italie du seizième siècle», Annales ESC, vol. 46, n 4 , juillet-août I991, p. 789-805.

90. Olivier Bouquet, "L'autobiographie par l'État sous les derniers Ottomans», Turcica, vol. XXXVIII, 2006, p. 25I-279. 
Grand Seigneur), serviteurs soumis à son autorité (Ibrahim Pacha baisant la robe de Soliman), tombés courageusement au combat (Hüseyin Pacha), ou exécutés pour être entrés en rébellion (Ahmed Pacha) ${ }^{91}$. L'introduction de la photographie prolongea cette tradition des Ottomans aux côtés de l'Ottoman : les dignitaires figuraient très souvent en couverture des revues officielles, au même titre que les réalisations impériales de tous ordres (écoles, casernes, hôpitaux). La photographie était un instrument de publicisation du pouvoir, dans le cadre des expositions universelles par exemple. Il en était de même des héros du régime et des réformateurs de l'Empire. À travers eux, c'était la capacité de l'Empire à survivre et à se réformer qui était opposée à des observateurs sceptiques et des puissances hostiles. Mais si le pouvoir jugeait utile de les inscrire dans une mise en scène, un cérémonial ottoman, eux-mêmes tardaient à se faire portraiturer sous leurs meilleurs atours.

On sait bien que la représentation humaine est problématique en terre d'islam et qu'elle posait problème aux Ottomans. Evliya Çelebi rapporte qu'au milieu du XVII ${ }^{e}$ siècle un janissaire avait détruit tous les portraits d'un Shahnâme dont il avait fait l'acquisition, convaincu que les miniatures étaient interdites ${ }^{92}$. Mais cet événement était assez iconoclaste pour être mentionné par le chroniqueur. Sans compter qu'aux dires de Mouradgea d'Ohsson, même «les chrétiens n' [avaient] ni le goût des tableaux, ni l'habitude de se faire peindre ${ }^{93}$; et que l'interdit fut largement levé par les souverains ${ }^{94}$; eux-mêmes s'entouraient des portraits de leurs prédécesseurs, notait ce même auteur au sujet d'Abdulhamid $I^{\text {er. }}$. On en revient à cette différence entre sultans et serviteurs. Les premiers avaient d'impérieuses raisons de vouloir se représenter et de représenter leurs dignitaires : on a évoqué plus haut l'impératif de légitimation qui pesait sur la maison d'Osman dans les provinces arabes ; était également en jeu - l'historiographie l’a bien établi - la défense d'une continuité dynastique insérée dans une vision cosmogonique et une permanence d'ordre divin (de Noé aux compagnons du Prophète) ${ }^{95}$. Il est vrai

9I. Feridun Emecen, «İbrâhim Pasa, Makbul», dans Diyanet Vakfı İslam Ansiklopedisi, vol. 2I, 1989, p. 333-335 (Süleymannâme, Topkapı Sarayı Müzesi Koleksiyonu, Hazine nr. I517, vr. I89, 374). On trouvera de magnifiques reproductions de ces scènes dans Esin Atıl, Süleymaname. The Illustrated History of Süleyman the Magnificent, New York, Harry N. Abrams, 1986.

92. "When the witty fellow brought [the Shanâme] to his tent and began leafing through, he saw that it contained miniatures. Painting being forbidden according to his belief, he took his Turkish knife and and scraped the narcissus eyes of those depicted, as though he were poking out their eyes, and thus he poked holes in all the pages. Or else he drew lines over their throats, claiming that he had throttled them" (Robert Dankoff, Evliya Çelebi in Bitlis, Leiden, Brill, I990, p. 294-295).

93. Mouradgea d'Ohsson, Tableau..., op. cit., tome 4, p. 274.

94. Selon Mouradgea d'Ohsson, les premiers souverains, Osman et Orhan, auraient commandé leur portrait. C'est inexact : le premier portrait est celui de Mehmed II, et la tradition débute vraiment dans les années I570.

95. Colin Imber, «The Ottoman Dynastic Myth», Turcica, 1987, vol. XIX, p. I6-I8 ; Gülrü Necipoğlu, "The Serial portraits...", art. cit., p. 50-54. Les Ottomans se réclament de Japheth, le fils de Noé. Dans une autre tradition, un lien avec Adam est proposé, toujours d'actualité dans certains almanachs officiels de la seconde moitié du XIx siècle (on trouvera une généalogie florale descendante, qui relie, en vingt-et-un tableaux, Adam au souverain régnant Abdülhamid II, dans un almanach de gouvernorat général : Salname-i Vilayet-i Hüdavendigâr, Bursa, matbaa-i vilayet, 
qu'après le $\mathrm{XVI}^{\mathrm{e}}$ siècle la dynastie s'estimait à ce point légitime qu'elle ne ressentait plus le besoin de s'inventer des origines comme aux premiers temps de son règne. Mais si l'«image publique de l'État» (S. Deringil) obéissait à d'autres nécessités et trouvait d'autres modes d'expression ${ }^{96}$, les dignitaires n'avaient toujours pas les moyens de se l'approprier. Tout au plus, certains d'entre eux, au gré des hasards et des inclinations, et à titre privé, profitaient-ils de leur proximité avec les cours princières ou leurs représentants, pour se faire croquer, tels les grands vizirs Mehmed Sokollu Pacha et Halil Hamid Pacha ${ }^{97}$. Ils étaient certes plus nombreux, au XIX ${ }^{\mathrm{e}}$ siècle, à accrocher leur portrait dans leur résidence ( $\mathrm{yal}$ ), ou à envoyer des photographies à leurs proches - comme bien d'autres Ottomans du reste. Mais s'ils se représentaient davantage individuellement, ils tardaient à mettre leurs pas dans ceux du sultan qui les avait devancés dans des temps plus anciens : ils n’avaient toujours pas la passion généalogique.

La généalogie existait, mais dans des cadres différents de ceux dont on a parlé, et auxquels on ne pense pas au premier chef : les arbres étaient aussi des instruments au service de la gestion des ressources familiales, des instruments d'enregistrement et de classification des ayants-droits, conçus par les administrateurs et les bénéficiaires de fondations pieuses. Nous en venons à l'examen de nos hypothèses.

\section{La généalogie par le vakıf}

\section{Le vakif, affaire de famille}

S'il y a une source mobilisée ces dernières décennies par les ottomanistes désireux de voir les familles de plus près, c'est bien les documents des vakıf(pl. : evkaf). Ils ont fait l'objet d'une littérature scientifique remarquable, au cœur des évolutions historiographiques de la discipline : histoire des notables, histoire urbaine, histoire économique, histoire sociale, ou histoire religieuse se sont relayées pour en exploiter les fonds considérables ${ }^{98}$. Parmi ces documents, les vakfye occupent

I303 H., p. I03-I23 ; voir également Selim Deringil, «The Invention of Tradition as Public Image in the late Ottoman Empire, I808-1908", Comparative Studies in Society and History, vol. 35, $\mathrm{n}^{\circ} \mathrm{I}$, jan. 1993, p. 3-29). Notons cependant que les Ottomans n'invoquaient pas de lien direct avec le Prophète. Dans une histoire épique, le Düsturnâme, l'historiographe Enveri retrace la généalogie ottomane jusqu'aux compagnons du Prophète, mais cette proposition reste isolée. Plus utilisé est le rattachement au clan Qayi, soi-disant issu d'Oguz Khan, qui aurait conquis la planète et donné naissance à vingt-quatre tribus. Cette version devient ensuite la généalogie officielle (Hasan Karateke, "Legitimizing the Ottoman Sultanate : A Framework for Historical Analysis", dans Hasan Karateke et Maurus Reinkowski (dir.), Legitimizing the Order. The Ottoman Rhetoric of State Power, Leiden, Brill, 2005, p. 23-24).

96. Sur la politique de l'image dynastique et ses outils héraldiques et musicaux, voir Selim Deringil, "The Invention... », art. cit., p. 4-7.

97. Julian Raby, «From Europe...", art. cit., p. I62 ; İsmail Hakkı Uzunçarşılı, "Sadrazam Halil Hamid Paşa», Türkiyat Mecmuast, vol. V, 1935, p. 215.

98. Le programme fixé par Fuad Köprülü en 1938 à partir des documents des vakıfa été en grande partie mis en œuvre ("L'institution du Vakouf. Sa nature juridique et son évolution historique», Vakıflar Dergisi, vol. II, 1942, p. 5). Cela dit, il n'était pas alors question d'histoire sociale ou 
une place centrale. On appelle ainsi l'acte juridique par lequel le fondateur définit la vocation du vakıf, en détermine les modalités d'administration (tevliyet)99, c'est-à-dire les fonctions et les rémunérations des employés, et en confie la charge à un administrateur (mütevelli). Conformément au droit hanéfite en vigueur dans la plupart des domaines impériaux, celui-ci pouvait très bien être un proche, un client, un esclave affranchi, voire un non-musulman ${ }^{\text {100 }}$. Cependant, il arrivait fréquemment que l'administrateur fût le fondateur lui-même, sinon un membre de sa famille, et qu'il optât pour ce que Yediyıldız a identifié pour le XvıII siècle comme "un vakif semi-familial». Cette formule permettait en effet de financer à long terme des œuvres charitables, tout en assurant des revenus permanents aux membres de sa famille ${ }^{\text {ror }}$. Cet intérêt, bien des fondateurs l'avaient compris : les trois-quarts des vakıf $\mathrm{du}$ XviII ${ }^{\mathrm{e}}$ siècle relevaient de ce régime. Il faut dire que ce "paravent» (pour reprendre l'expression de Jean-Claude Garcin ${ }^{102}$ ), adapté du vakıf de bienfaisance, permettait d'immobiliser l'essentiel d'une fortune sous une forme inaliénable, d'entretenir sa postérité, et d'assurer l'avenir de ses descendants dans le cas où ses biens seraient saisis, en vertu de la pratique de la confiscation, dite müsadere, qui frappait grand nombre de dignitaires civils et militaires. Une partie des biens était confisquée et mise aux enchères - généralement les fermes, les monnaies, les objets de prix et les équipements militaires, alors que les biens immobiliers étaient laissés aux héritiers ${ }^{\mathrm{TO}}$. De facto, les vakıf demeuraient ; ils continuaient d'être administrés, soit par le même homme, soit par un descendant du fondateur ${ }^{104}$. C'était l'usage, et le droit l'autorisait : l'administrateur devait être majeur, c'est-à-dire arrivé à maturité physique ${ }^{\mathrm{ros}}$. Il fallait en outre qu'il fût doué de raison. En théorie, il devait même être le plus apte des enfants (evladımın erşedi,

d'histoire de la famille ; et en effet, ce sont les dimensions les moins (ou les plus récemment) explorées. La bibliographie sur les vakıf est trop riche pour que j'en fasse référence ici. On en trouvera quelques éléments, surtout pour les provinces arabes, dans Randi Deguilhem, «Wakf. IV. Dans l'Empire ottoman jusqu'en I9I4", EI ${ }^{2}$, Leiden, vol. XI, 2005, p. 95-IOI.

99. Bahaeddin Yediyıldız, L'Institution du Vaqf au XVIIT siècle en Turquie. Étude socio-historique, Ankara, Éditions du ministère de la Culture, 1990, p. I48.

Ioo.Ahmet Akgündüz, İslâm Hukukunda ve Osmanlı Tatbikatında VakıfMüessesesi, Istanbul, Osmanlı Araştırmaları Vakfı, 1996, p. 319 ; Nazif Öztürk, «Mütevelli», Diyanet Vakfı Íslam Ansiklopedisi, vol. 32, 2006, p. 217-220. Dans l'échantillon proposé par Yediyıldız, les premiers administrateurs d'une grande partie des vakıf du XviII ${ }^{\mathrm{e}}$ siècle (5I \%) sont les fondateurs eux-mêmes. 47 \% transmettent cette fonction à leurs enfants, et, après leur extinction, à leurs proches parents (37\%) ou à leurs esclaves affranchis (Io \%) (Bahaeddin Yediyıldız, L'Institution..., op. cit., p. I48-I50).

IOI.Pour situer cette forme juridique entre les formes classiques dites waqf hayrî et waqf dhurrî, voir notamment John R. Barnes, An Introduction to Religious Foundations in the Ottoman Empire, Leiden, Brill, 1986.

IO2.Jean-Claude Garcin, "Le waqf est-il la transmission d'un patrimoine ?», dans Joëlle Beaucamp et Gilbert Dagron (dir.), La transmission du patrimoine. Byzance et l'aire méditerranéenne. Travaux et mémoires du Centre de Recherche d'Histoire et Civilisation de Byzance, Paris, De Boccard, 1998, p. IO2.

I03. Fatma Müge Göçek, «Musâdara. 3", EI², vol. VII, 1992, p. 652-653.

I04.De fait, lorsqu'un administrateur vient à décéder et qu'il a été le fondateur du vakıf, c'est toujours l'un des enfants, sinon un tiers, qui prend la succession (Bahaeddin Yediyıldız, L'Institution..., op. cit., p. 150).

I05. Ahmet Akgündüz, İslâm Hukukunda..., op. cit., p. 314-315. 
ou en iyi yönetebilecek olan, pour reprendre la terminologie des vakfiye ${ }^{106}$, doté d'un niveau suffisant de sagacité, de jugement et d'intégrité. Une fois cet administrateur disparu, un autre lui succédait. Il s'agissait très souvent d'un autre membre de la famille, «de génération en génération» selon la formule (batnen bade batnin), et ainsi de suite ("aux enfants et aux enfants des enfants»: evlad ve evlad-i evlad).

L'administrateur était notamment chargé de la répartition des revenus de la fondation selon les bénéficiaires. Dans le cas où ceux-là étaient des descendants du fondateur, il lui fallait les identifier, génération après génération. Le meilleur moyen de le faire était de recourir à un arbre généalogique. Comme les vakıf s'étaient multipliés, surtout au XVIII ${ }^{\mathrm{e}}$ siècle, ils furent à l'origine de nombreux şecere que les familles constituaient, actualisaient et transmettaient. On en trouve encore aujourd'hui, car certains de ces vakif continuent d'exister. Nous allons prendre l'exemple de l'un d'entre eux.

\section{Vakif d'un grand vizir, arbre d'une grande famille}

À la fin du mois de mai 1785, le grand vizir Halil Hamid Pacha fut exécuté sur ordre du sultan. Les nombreuses fermes (malikane) en sa possession furent mises aux enchères quelques jours après que la nouvelle de son exécution fut rendue officielle et que sa tête fut exposée. Le trésor impérial put ainsi s'octroyer la somme considérable de 300000 piastres. Mais il ne put mettre la main sur l'essentiel de la fortune du dignitaire : il était d'usage de laisser à la famille les terres et les maisons. Surtout, à l'instar de ses prédécesseurs - on cite souvent l'exemple inégalé du précité Ibrahim Pacha -, Halil Hamid Pacha avait investi ses profits dans le financement et l'administration de toutes sortes de fontaines, mosquées, couvents et bibliothèques. Nous ne citerons ici que les fondations établies à Isparta, pour la raison qu'il s'agissait de sa ville de naissance, et qu'à l'évidence, il la considéra comme un lieu privilégié pour y imprimer sa marque - il y a certainement, chez ce grand vizir non-issu du devsirme ${ }^{107}$, un ancrage de l'imaginaire ou de la postérité qui mériterait d'être davantage creusé. Il fit construire un aqueduc; il fit également restaurer, élargir les ailes, et ajouter un minaret à la mosquée İplik Pazarı (ou İplikçi), construite par un notable de la ville, Hacı Abdi Ağa dans les années $1560^{108}$. Il adjoignit à cette mosquée (qu’il fit restaurer) une bibliothèque dans un bâtiment nouvellement construit à cet effet, qu'il dota de 400 ouvrages, ainsi que deux fontaines. Nous disposons de la vakfiye de novembre $1783^{109}$; elle définit les modes d'administration des fondations d'Isparta, mais fixe aussi le devenir de l'ensemble des vakıf de Halil Hamid Pacha. À la mort du fondateur, son épouse devait en devenir l'administratrice. Puis à la mort de celle-ci, devait lui succéder l'aîné des enfants de la descendance (benim sulbi evladımın ekberi), puis, à l'épuisement de la génération, l'aîné des membres de la génération suivante, et

I06.Nazif Öztürk, «Mütevelli», art. cit., p. 217.

I07. «Ramassage» : système de recrutement de jeunes gens, originaires des Balkans ou d'Anatolie le plus souvent, destinés à l'armée ou aux services du Palais et de l'administration.

I08. La vakfyye, qui prévoit l'installation d'une bibliothèque dans un bâtiment en pierres, date de la fin

I783 (İsmail Erünsal, Osmanlı Vakıf Kütüphaneleri, Ankara, Türk Tarih Kurumu, 2008, p. 249). I09. Vakıf Genel Müdürlü̈̆̈̈ Arşivleri (Ankara, registre n 628, p. 547, n 289). 
ainsi de suite. En cas d'interruption de la descendance, l'administrateur devait être le plus proche des parents de la famille.

Tel ne fut pas le cas. Les descendants continuent aujourd'hui d'administrer les biens du vakıf familial. Et pour ce faire, ils disposent notamment d'un arbre généalogique. Nous ignorons au jour d'aujourd'hui quand celui-ci fut constitué. Dans la copie d'un arbre produit au $\mathrm{Xx}^{\mathrm{e}}$ siècle dont nous disposons, il est question d'un şecere mis en forme (tanzim ettiği şecere) en 1900 par Atıf Pacha, descendant de la quatrième génération. Dans cet arbre, les administrateurs successifs sont cités, à l'exception des deux premiers que nous retrouvons dans d'autres sources (l'épouse de Halil Hamid et son fils, Mehmed Arif Bey). Cet arbre présente un terminus a quo (I968), qui montre qu'il a été prolongé et actualisé régulièrement. Y sont indiqués plus de quatre cents descendants, autant de bénéficiaires de la fondation donc. Un tel nombre permet aisément de comprendre pourquoi l'arbre s'est imposé ; en quoi la famille a dû se mettre à la généalogie, à en maîtriser l'art. Car autant sur certains seçere de la période ottomane - ceux des Cemali par exemple ${ }^{\text {IIO }}-$, les descendants n'étaient pas encore assez nombreux pour avoir à lier les noms entre eux de manière formelle, autant dans le cas d'aussi grandes familles, il était hors de question de ne pas introduire une certaine rigueur, dès lors qu'il s'agissait d'assurer une juste répartition des revenus de la fondation, surtout dans le cas où il s'agissait de gros sous ${ }^{\text {III }}$.

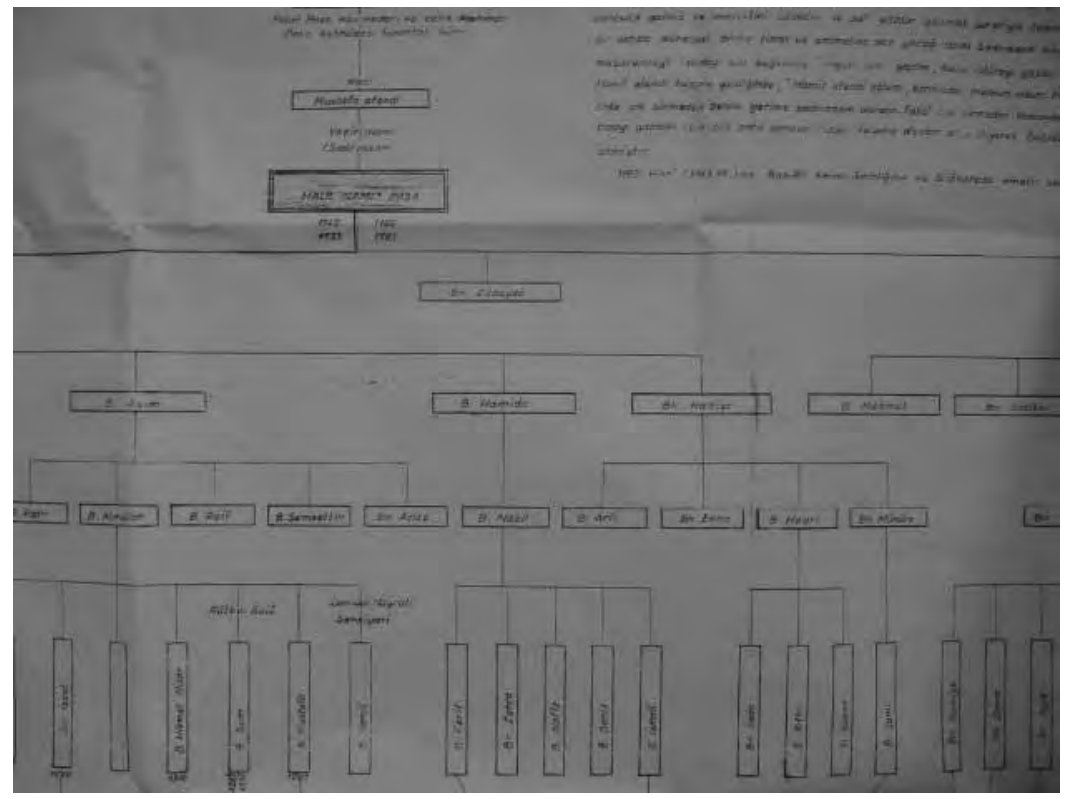

Une partie du şecere des descendants de Halil Hamid Pacha

IIo. Yusuf Küçükdağ, II. Bayezid..., op. cit.

III. Voilà qui rappelle les systèmes généalogiques des premiers temps de l'islam, conçus dans le cadre du système de distribution des pensions civiles (Claude Cahen, "Atầ ", EI², vol. I, p. 75075I). À ce sujet, voir aussi Jacqueline Sublet, Le Voile du nom. Essai sur le nom propre arabe, Paris, PUF, 199I, p. 22-23. 
En effet, la rigueur de la présentation de la généalogie frappe le lecteur. Le document se présente sur une feuille papier rectangulaire de $165 / 67 \mathrm{~cm}^{112}$. Il est manuscrit ; il est lisible pour l'essentiel. L'ensemble est remarquablement ordonnancé. L'espace du document est bien exploité. L'auteur définit le document comme un şecere. Les spécialistes de généalogie diraient, eux, qu'il s'agit d'un diagramme : des noms propres sont notés dans des rectangles; ils sont liés les uns aux autres par des lignes tracées à la règle, dont la plupart indiquent des liens de filiation ; à l'exception d'une branche collatérale et d'une dizaine d'alliés, tous les individus sont reliés à un même ascendant, également indiqué dans le titre du document : le grand vizir Halil Hamid Pacha. L'auteur de l'arbre, Cemal Bükey, général de formation, a introduit comme une rigueur toute militaire dans la disposition des noms ; peut-être a-t-il mobilisé ses compétences logistiques et les services de l'administration qui l'employait ; peut-être a-t-il mis en application un certain goût pour la généalogie ; peut-être est-il tombé sur des manuels spécialisés - cela dit, on ne devait pas les trouver sous le sabot d'un cheval dans la Turquie républicaine du milieu du $\mathrm{xx}^{\mathrm{e}}$ siècle.

Un dernier mot, avant de passer à d'autres formes généalogiques et d'en venir aux continuités entre les périodes ottomanes et républicaines. Si notre hypothèse est que l'arbre n'avait été constitué par la famille que dès lors qu'il y avait quelque chose à transmettre en son sein, l'objet de la transmission ne se réduisait pas à un capital matériel ou immobilier - c'est ce que suggère par exemple une comparaison avec la baraka acquise comme descendant du Prophète et/ou de tel saint, comme héritier (khalîfa) de tel cheikh, et qui donnait le droit de prendre la tête, entre autres, de la zâwiya et des disciples ${ }^{113}$. Certainement y avait-il dans cette logique juridique de la fondation pieuse de quoi alimenter des formes de solidarités entre les descendants autres que matérielles, un sentiment d'appartenance et d'identification à un même ensemble, inscrit dans une même référence ancestrale, selon trois dimensions plus ou moins successives, plus ou moins imbriquées : Halil Hamid comme ancêtre fondateur, patrimonialiste dès les premières générations ; éponyme dès les suivantes (les Halil Hamid Paşa-zâde), selon la transformation du patronyme en nom de famille en -zâde ${ }^{\mathrm{II} 4}$; mémoriel, certainement avant (mais de toute façon après) la loi qui imposa l'obligation du nom de famille en 1934. Nous touchons ici au sujet de l'ancestralité auquel nous consacrerons une autre étude. Son évocation nous permet ici d'introduire notre seconde hypothèse.

II2. Taha Toros a eu la générosité de m’en donner une copie. Je l'en remercie chaleureusement.

II3. Sur la transmission héréditaire du charisme, voir Catherine Mayeur-Jaouen, "Le Saint musulman...", art. cit.

II4. Sur ces formes en -zâde, voir Olivier Bouquet, "Onomasticon Ottomanicum : identification administrative et désignation sociale dans l'État ottoman du XIX siècle", Revue des mondes musulmans et de la Méditerranée, $\mathrm{n}^{\circ}$ 127, 2010, p. 213-234. 


\section{Sous la République : le retour des 0 ttomans}

\section{Les généalogies à l'honneur}

Si l'on fait l'état des lieux de la production généalogique de la période républicaine, on note que les arbres cités sont tantôt des documents anciens reproduits et actualisés, tantôt des diagrammes constitués à l'occasion de publications, à partir

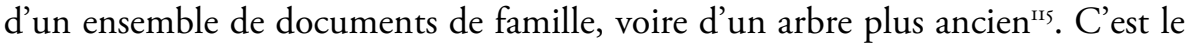
cas de la généalogie précitée : Cemal Bükey s'est inspiré d'un diagramme mis en forme en 1900 par son oncle patrilinéaire feu Atıf Pacha, puis authentifié (tasdik) en 1922 par un autre parent, Mehmed Salih Bey. Il a mobilisé divers papiers de famille, notamment ceux laissés par son père, Derviş Pacha. Puis il l'a complété à l'aide d'études historiques qu'il cite, et très probablement, d'échanges avec d'autres membres de la famille. Cet exemple invite à faire la différence entre la mémoire réflexive des familles et la formalisation généalogique qui en procède. La première a été entretenue au long des âges ottomans à la fois par les descendants et, pour ce qui est des plus célèbres d'entre elles, par les chroniqueurs. La seconde est souvent plus récente, organisée à l'occasion d'une publication ou d'une commémoration, comme la généalogie de la famille Okday par exemple, produite par l'un des petits-fils du dernier grand vizir Ahmed Tevfik Pacha ${ }^{\text {II }}$. Dans bien des cas, la mémoire de l'auteur suffit à nourrir la mise en forme de l'arbre ${ }^{\mathrm{II}}$. Dans le cas contraire, on trouve encore des érudits en Turquie qui ont constitué leurs propres diagrammes et qui complètent leurs informations à l'aide de répertoires - celui d'Öztuna est le plus connu ${ }^{\text {II8 }}$.

Le genre généalogique se développe depuis quelques décennies. Des arbres sont publiés dans des études scientifiques ${ }^{\text {II) }}$; d'autres, dans des mémoires ou des

II5. Malmîsanij reconstitue la généalogie des Bedirhan à l'aide d'arbres manuscrits que lui ont communiqués deux descendants, et qu'il complète à l'aide d'autres sources - la bibliographie citée est très riche (Cızira Botanlı Bedirhaniler ve Bedirhan Ailesi Derneğinin Tutanakları, Istanbul, Avesta, 2000, p. 86-96).

II6. Şefik Okday, Büyükbabam Son Sadrazam Ahmet Tevfik Paşa, Istanbul, Sebil Yayınevi, 1986, p. I45-I46. La généalogie est clairement organisée par générations, classées en quatre groupes de naissance (I843-1859 ; I88I-I888 ; I906-I9I7 ; I938-1956). L'objet n'est pas de restituer l'ensemble de la descendance : ne figurent ni les jeunes qui ne sont pas encore sur le marché du travail, ni les «femmes au foyer» disparues sans avoir laissé d'enfants. En revanche, les professions - souvent prestigieuses - sont indiquées. Il s'agit bel et bien, pour l'auteur, de mettre en valeur la reconversion fonctionnelle de la famille au service de la République.

II7. Deux descendantes de Şakir Paşa ne semblent avoir recouru qu'à leurs souvenirs et papiers personnels, dans leurs mémoires respectifs. Les contradictions et imprécisions sont multiples : Şirin Devrim, Şakir Paşa Ailesi "Harika Çılgınlar», Istanbul, Milliyet kitapları, 1997 ( 3 éd., traduit de l'anglais), p. 9 ; Nermidil Erner Binark, Şakir Paşa Köşkü, Istanbul, Remzi, 200o, p. 7. II8. Yılmaz Öztuna, Devletler ve Hânedanlar. Türkiye (I074-I99o), Ankara, Kültür Bakanlığı Yay., 1996 ( $2^{\mathrm{e} e ́ d .) . ~}$

II9. İsmail H. Uzunçarşıll, Meşhur Rumeli Âanlarından Tirsinikli İsmail, Yılık Oğlu Suleyman Ağalar ve Alemdar Mustafa Paşa, Istanbul, Maarif Matbaası, I942, p. 194-I95 (il est fait référence à un arbre familial) ; İsmail Hakkı Uzunçarşılı, «Tunus'un I88I'de Fransa Tarafından İşgaline Kadar Burada Valilik Eden Hüseynî Ailesi ", Belleten, vol. XVIII, nº 72, 1954, p. 545-580; İsmail H. Uzunçarşılı, Çandarlı Vezir Ailesi, Ankara, Türk Tarih Kurumu, 1988 (rééd.) ; Mithat Cemal Kuntay, Namık Kemal Devrinin İnsanları ve Olayları Arasında, vol. I, Istanbul, Maarrif Matbaası, 
biographies. Certains d'entre eux respectent les règles de représentation des traités généalogiques ${ }^{120}$, d'autres recourent à des formes plus aléatoires ${ }^{121}$. Il en est même qui sont reproduits sur des sites internet de vulgarisation ${ }^{122}$. Les Turcs sont aujourd'hui engagés, comme d'autres peuples, dans la recherche mémorielle des origines. Il y a chez eux comme un goût retrouvé pour le passé impérial, pour ses arts, pour la restauration de ses yalı, pour ses modes de fondation : les sociétés constituent des vakıf qui rappellent ceux dont nous parlions. Des réunions de famille sont organisées entre descendants - l'exemple des Evrenos a été récemment évoqué $^{123}$; on se retrouve à l'occasion de commémorations, comme celle de la fondation Cemali-i Aksaray. Des débats sur les origines du nom de famille sont tenus. Des familles s'accusent mutuellement d'usurpation; un cas emblématique est celui qui oppose les descendants de Fuad Köprülü et les Köprülü d'Izmir qui se disputent le rattachement à la prestigieuse lignée des grands vizirs. Le contexte politique est favorable au retour des Ottomans : le régime actuel ne voit plus d'un si mauvais œil qu'à l'époque kémaliste les hautes figures impériales ${ }^{124}$ : écrivains et historiens n'hésitent pas à rappeler qu'ils sont les descendants de personnages peu appréciés des fondateurs du régime ${ }^{\mathrm{r2}}$. La presse a le goût des rapprochements entre les grands hommes du temps et leurs ancêtres de la réforme ottomane : pour revenir à notre famille, entre Kemal Derviş, ministre de l'Économie, célébré par la presse pour avoir tiré la Turquie de la crise financière de 200I, et Halil Hamid Pacha, présenté par l'historiographie classique et récente comme l'un des meilleurs grands vizirs de la période moderne ${ }^{\mathrm{r2}}$.

Nuançons aussitôt : cette mode généalogique, cet «embourgeoisement de la mémoire», (pour reprendre les termes d'André Burguière ${ }^{\mathrm{I27}}$ ) n’a rien de la passion

I944, p. 387 ("soy kütüğü» (arbre généalogique), de Reşat Bey (Pacha), dessiné par sa fille Ubeyde Fergar) ; Stephen Hemsley Longrigg, Four Centuries of Modern Iraq, Beyrouth, Librairie du Liban, I968, p. 348 ; Necdet Sakaoğlu, Anadolu Derebeyi Ocaklarından Köse Paşa Hanedanı, Istanbul, Tarih Vakfi, I998, p. 29I.

I20. On trouvera un arbre généalogique des cinq derniers souverains en trois tables, bien présentées, proches du système de descendance en escalier (mais sans sigles), dans l'ouvrage du journaliste Yılmaz Çetiner consacré au sultan Vahideddin et à ses descendants (Son Padişah Vahideddin, Istanbul, Epsilon, 2005).

I2I. Ali Ekrem Bolayır, Hâtıralar, Ankara, Hece Yay., 2007, p. 430-43I (arbre de la prestigieuse famille de Namık Kemal sur une même page, mais avec un système aléatoire de citation des noms).

I22. L’arbre de la célèbre famille arménienne, les Balyan : http ://tr.wikipedia.org/wiki/Balyan_Ailesi (consulté le 22/II/20IO).

123. Heath Lowry et İsmail Erünsal, "The Evrenos... », art. cit.

124. Olivier Bouquet, "Maintien et reconversion des noblesses ottomanes aux débuts de la République turque", Vingtième Siècle. Revue d'histoire, n 99, juillet-sept. 2008, p. I29-142.

I25. Sinan Kuneralp, sur son grand-père Ali Kemal Bey : «Bir Osmanlı aydının gözüyle Fransız ihtilali : Ali Kemal'in Rical-i İhtilâl'i", dans 200 Yuldöneminde Fransız İhtilâli ve Türkiye Sempozyumda Sunulan Bildiriler, Konya, Selçuk Üniversitesi, 1991 ; Son Dönem Osmanl Erkân ve Ricali (I839-I922). Prosopografik Rehber, Istanbul, İsis, I999, p. XI.

I26. İsmail H. Uzunçarsılı, "Sadrazam Halil Hamid», art. cit., p. 245 ; Virginia H. Aksan, An Ottoman Stateman in War and Peace: Ahmed Resmi Efendi, $1700-1783$, Leiden, Brill, 1995, p. XVIII ; Fikret Sarıcaoğlu, Kendi Kaleminde Bir Padişahın Portresi : I. Abdülhamid (I774-I789), Istanbul, Tarih ve Tabiat Vakfi Yayınları, 200I, p. I89, 198, 264.

I27. André Burguière, «La mémoire familiale du bourgeois gentilhomme : généalogies domestiques 
qui a embrasé les Européens occidentaux ces dernières décennies ${ }^{128}$. On trouve peu de clubs de généalogistes sur internet ${ }^{129}$; peu de généalogistes en herbe, comme ceux qui peuplent les archives régionales chez nous. Le répertoire des grandes familles le plus utilisé ne présente aucune généalogie ${ }^{130}$. La plupart des livres consacrés aux familles - sujet qui se développe sans atteindre des niveaux de production remarquables - en sont dépourvus. Les noms des membres de la famille sont souvent cités les uns à la suite des autres, un peu comme les nasab, sans que l'auteur se soucie toujours de savoir si le lecteur s'y retrouve ${ }^{131}$. Le discours historique sur la famille reste à la fois anecdotique, relié aux grands événements et aux grands hommes, et compilatoire. Il livre des photographies individuelles plus que des représentations d'ensemble.

Je n'ai pas encore assez fait le tour de la production pour proposer une typologie fine des adeptes de la généalogie. Mais je crois repérer que la pratique est davantage liée à des groupes spécifiques quà des citoyens venus de tous les horizons. Puisque c'est la famille impériale qui a donné au genre généalogique une dimension qu'il n'avait pas avant elle, autant commencer par elle.

\section{Anoblissement de la mémoire et culture diasporique}

Nous avions quitté les Ottomans piètres généalogistes. Nous les retrouvons à la fin du $\mathrm{xx}^{\mathrm{e}}$ siècle en adeptes chevronnés de la pratique. Ils prennent l'affaire très au sérieux. En 1995, ils ont créé une association à Paris ${ }^{132}$. Ils publient les mémoires laissés par leurs parents, inventorient et classent les descendants (généralement par sultan) selon les statuts distingués plus haut ${ }^{133}$. Ils connaissent la position de chacun sur l'arbre de la famille, avant même de se rencontrer, tous, et ce pour la première fois, en septembre $2006^{134}$. Des journalistes écrivent des livres à leur sujet qui font parler d'eux ${ }^{135}$. Le fait n'est paradoxal qu'au premier abord :

en France aux XVII et XVIII ${ }^{\mathrm{e}}$ siècles", Annales ESC, vol. 46, n 4, I991, p. 782.

I28. Sur la généalogie buissonière et les dérives du genre, voir Martine Segalen et Claude Michelat, "L'amour de la généalogie», dans Martine Segalen (dir.), Jeux de famille, Paris, CNRS, I99I, p. I93-208; Sylvie Sagnes, "De terre et de sang : la passion généalogique», Terrain, n 25, I995, p. $125-\mathrm{I} 46$.

I29. http ://www.soyagaci.gen.tr ; http ://www.secere.org (consultés le 20/o9/2009).

I30. Yilmaz Öztuna, Devletler..., op. cit.

I3I. Ahmet Nuri Sinaplı, Şeyhül Vüzera, Serasker Mehmet Namık Paşa, Istanbul, Yenilik Basımevi, 1987, p. 289-296 ; Abdullah Muradoğlu, Reformun Dervişleri. Halil Hamit Paşa'dan Kemal Derviş̧e. Bir Ailenin Biyografisi, Istanbul, Bakış, 200I.

I32. Hanedan, Maison d'Osman : siège social : Io rue Edmond Guillout, 750I5 Paris. Son objet officiel est la "défense devant toute juridiction de l'honneur et des intérêts individuels et collectifs des descendants ottomans issus du sultan Osman $\mathrm{I}^{\mathrm{er}}$ " (Journal officiel, $\mathrm{n}^{\circ}$ de parution 200300I7) http ://www.journal-officiel.gouv.fr/association/index (consulté le 20/10/2009).

133. Osman Osmanoğlu, Ali Vâsıb Efendi..., op. cit. (avec des données chiffrées et généalogiques très complètes sur le hanedan, p. 413-456).

134. Kerime Senyücel, Hanedan..., op. cit., p. I2.

135. Murat Bardakçı est celui qui s'est le plus illustré dans l'hagiographie des derniers Ottomans : Son Osmanlılar : Osmanlı Hanedanının Sürgün ve Miras Öyküsü, Istanbul, Pan Yayınc1lı, I99I (avec un şecere non exaustif et des explications sur les difficultés de l'auteur à représenter un arbre d'un seul tenant, p. 2II-2I9) ; Murat Bardakçı, Şabbaba, Istanbul, Gri, I998 (avec un arbre 
la famille ottomane vit en exil, dispersée dans plusieurs pays, alliée à d'autres familles princières déchues ou régnantes ${ }^{136}$; mais l'État n’est plus là pour assurer la continuité de son statut ; elle est définitivement privée des moyens de se concevoir en dynastie politique; c'est alors qu'elle s'affiche comme noblesse, comme une lignée dont tous les rameaux tirent un prestige semblable de l'ascendance ottomane, laquelle non seulement est ancienne, puisqu'elle remonte à Ertuğrul, donc à la seconde moitié du XIII ${ }^{\mathrm{e}}$ siècle, mais reconnue comme ininterrompue : la continuité de l'État ottoman l'atteste. "Anoblissement de la mémoire» pour le coup, cette culture généalogique entretenue prend les formes d'une culture d'exil, d'une culture de diaspora même. C'est la marque d'une famille qui répond, dans un contexte de globalisation, aux craintes de la dispersion par la mobilisation mémorielle.

Le fait est singulier. Acteur de l'histoire devenu objet mémoriel, l'Empire ottoman est l'espace de référence d'une culture diasporique, après avoir été le lieu d'accueil de diasporas ${ }^{137}$. On note en effet des pratiques généalogiques comparables chez quelques familles immigrées au XIX ${ }^{\mathrm{e}}$ siècle. Deux cas, parmi d'autres, l'illustrent.

Le premier concerne ces quelques familles, chassées d'Europe centrale et orientale à la suite des révolutions de la première moitié du XIX ${ }^{e}$ siècle. Souvent de petite et moyenne noblesse polonaise, croate ou hongroise, sans doute ont-elles poursuivi sur les bords du Bosphore des modes de solidarité et de récit de soi déjà explorés sur les rives du Danube ou de la Sava. Mais elles ont aussi créé de nouveaux liens à Istanbul, par le jeu des alliances et la proximité géographique : un auteur populaire démêle les liens entre quatre d'entre elles, en qui il voit une "tribu du Bosphore» (Boğaz'daki aşirett) ${ }^{138}$, agrégat de familles chrétiennes progressivement intégrée à la nation, par des alliances avec des familles productrices de hautes figures nationales (Namık Kemal par exemple), par la production d'un discours ethno-généalogique, tels celui de Mustafa Celaleddin qui, dans un livre publié à la fin des années i860 (Les Turcs anciens et modernes), défend la thèse d'une origine aryenne des Turcs d'Europe, une thèse qui aurait intéressé Mustafa Kemal lui-même ${ }^{139}$. Il y a là sans doute une contribution non reconnue par l'idéologie

généalogique de la descendance de Sultan Mehmed Vahideddin, p. 677). Il est désormais facile de se procurer des représentations généalogiques des sultans dans les éditions populaires : voir Abdülkadir Dedeoğlu, Album of the Ottomans, Istanbul, Osmanlı Publishing House, 1982, p. 6-Io. 136. Ainsi Dürrüşehvar, la fille du calife Abdülmecid, épouse-t-elle en I93I le prince de Berar (Anthony Alderson, The Structure..., op. cit., p. 89) ; Hanzade Sultan et Neslişah Sultan épousent des princes égyptiens en 1940 (Osmanoğlu, Ali Vâsıb Efendi..., op. cit., p. 307-308) ; en 1991, Ertuğrul Osman Efendi épouse, en secondes noces, une descendante de la famille royale afghane (Kerime Senyücel, Hanedan..., op. cit., p. 32). Sans oublier le cas, rendu célèbre par le roman de Kenizé Mourad (De la part de la princesse morte, Paris, Robert Laffont, 1987), du mariage de Selma Rauf avec le radjah de Baldapour.

137. Minna Rozen a récemment ouvert un espace de réflexion comparatif sur les diasporas juives et grecques dans le contexte ottoman et dans d'autres lieux de vie et de mémoire (Minna Rozen (dir.), Homelands and Diaspora. Greek, Jews and their Migrations, London, Tauris, 2008).

138. Mahmut Çetin, Boğaz'daki Aşiret, Istanbul, Edille, 1997.

139. Ibid., p. 19-20. 
officielle de la République turque, je veux parler d'un apport des familles nobles à la culture ethno-généalogique nationale, des formes d'auto-légitimation du rôle de l'autre, à la fois comme autre en exil, et comme assimilé en devenir. C'est aussi le cas du second exemple de familles à citer, les familles circassiennes implantées dans la seconde moitié du XIX ${ }^{e}$ siècle.

On en retrouve plusieurs munies d'arbres au lendemain de la seconde guerre mondiale. Dans les années 1960, et peut-être avant, les Tugay par exemple ont un arbre généalogique, ou plutôt une liste descendante de noms, sur le mode de certains annuaires familiaux. La reconstitution de la famille va de pair avec une réflexion sur leur origine tribale circassienne : l'un de leurs descendants le dit très explicitement dans le titre d'un article : "la tribu caucasienne Tuga et la famille Tugay». La proximité des noms (de famille et de tribu) est le point de départ de la réflexion. Quand on sait que l'arbre en question est tiré des papiers personnels du célèbre ambassadeur Ahmet Hulûsi Fuat Tugay ${ }^{140}$; quand on sait qu'il était le mari de Emine Fuad Tugay, issue de la famille princière égyptienne au sein de laquelle les reconstitutions généalogiques étaient alors monnaie courante, on pense à une possible influence entre deux pratiques généalogiques initialement distinctes.

Comment s'étonner alors que les descendants des dignitaires ottomans (qui désormais épousent aussi des membres de ces deux types de familles, anciennement immigrées, aujourd'hui parfaitement assimilées) ne versent pas à leur tour dans le genre généalogique, entre découverte d'une culture diasporique sur le mode de la famille impériale, et ancrage néo-ottoman distinctif dans la Turquie républicaine ? Prenons le cas des descendants de Halil Hamid Pacha. On en trouve aux États-Unis, en Allemagne, en France. Ils gardent pourtant le contact. Peut-être l'éloignement de la terre des ancêtres - de la Turquie-nation - nourrit-il entre les descendants une proximité relationnelle qui aurait été distendue s'ils étaient restés Stambouliotes ou Ankariotes. Peut-être s'agit-il seulement chez certains d'un tropisme passéiste pour une grandeur impériale disparue ? Peut-être est-ce davantage pour d'autres un regard tendu vers leur avenir, à l'échelle du monde ${ }^{\mathrm{r} 4 \mathrm{r}}$ ?

\section{Conclusion}

Cette étude visait à répondre à deux questions. Pourquoi les dignitaires ottomans pratiquèrent-ils peu la généalogie ? Pourquoi leurs descendants constituèrent-ils malgré tout des arbres et des tableaux de famille ? Pour la première question, j'ai procédé à un examen terminologique et historiographique. Pour la seconde, j'ai proposé mes propres hypothèses d'interprétation, en fonction des réponses apportées à la première question et des sources que j'ai consultées. Les résultats de l'enquête sont les suivants.

I40.Bedi N. Şehsuvaroğlu, Hekim Bir Siyasîmizin Portresi Büyükelçi. Dr. A. Hulûsi Fuad Tugay, Istanbul, Istanbul Matbaas1, 1972, p. 37-39.

I4I. À l'instar des familles bourgeoises européennes du Xvi ${ }^{\mathrm{e}}$ siècle (Martine Segalen et Claude Michelat, «L'amour de la généalogie... », art. cit., p. 195-196). 
Quand les sultans eurent assez de raisons pour entrer en généalogie, ils rejoignirent les nombreuses dynasties environnantes qui les avaient précédés dans la recherche de leurs origines et la glorification de leur continuité. Ils n'en eurent jamais assez, cependant, pour placer la généalogie au cœur de leur littérature et de leur historiographie; et leurs dignitaires encore moins pour investir le genre. Le système politique ottoman était ainsi : si les sultans gouvernaient des groupes socioculturels au sein desquels la généalogie avait son importance, les instruments principaux de leur pouvoir (les élites militaires et civiles en tout cas) n'étaient pas officiellement reconnus en tant que grandes familles. Si certains de ces hauts dignitaires se constituèrent malgré tout en "noblesse dans l'État» (reproduction des statuts, transfert des richesses aux descendants, transmission d'une culture d'État), ils n'eurent ni le droit ni les raisons de se dessiner des armes, de se représenter en généalogie, de se dire lignées ${ }^{142}$. Les dignitaires apprécièrent sans doute la qualité des "arbres à sultan", mais ils n’eurent jamais la passion de la généalogie. Comment expliquer alors que certains de leurs descendants en vinrent à se fabriquer des arbres et des tableaux?

Une première hypothèse d'explication est liée au système de transmission des biens dans le cadre des fondations pieuses. Il fallait recenser les individus, car il fallait organiser la juste et légale répartition des revenus entre les bénéficiaires. Une certaine historiographie laisse entendre que les vakıf, situés au cœur de l'économie du XVIII ${ }^{e}$ siècle, entrèrent en déshérence après que l'État en eut pris le contrôle au siècle suivant ; et qu'ainsi les fortunes familiales se constituèrent ailleurs, en vertu du Code de la propriété de 1858 , dans le cadre de la nouvelle économie capitaliste, ou à la faveur des privilèges patrimonialistes dont profitaient les dignitaires ${ }^{143}$. Même si les familles concernées n'ont aucun intérêt à le rappeler aujourd'hui, le fait est pourtant qu'une partie des vakıf subsistèrent, assurèrent le maintien de patrimoines, et la continuité de la mémoire des lignées. Chez certaines d'entre elles, la généalogie s'était dessinée au service de pratiques juridiques familiales élaborées au contact d'une culture islamique ancienne ; elle devint un outil gestionnaire au sein du vakıf, avant de se transformer en référent identitaire ; elle fut une infrastructure économique de la mémoire, avant d'être un appui symbolique du sentiment de la famille.

C'est là un second vecteur à prendre en compte : avec l'effondrement du système impérial, les Ottomans de la maison d'Osman (hanedan) et les Ottomans du service, jadis séparés par une barrière symbolique certes franchissable (un dignitaire pouvait devenir damad, gendre du sultan) mais réelle, connurent un destin commun : l'exclusion politique et l'exil. Pour les uns comme pour les autres, il y eut cette fois-ci une passion généalogique nourrie par l'auto-anoblissement des Ottomans : le hanedan n'avait pas la possibilité véritable de se penser comme famille royale; il devint une dynastie déchue. Les Ottomans n'avaient pas le droit de se penser comme aristocratie ; les néo-Ottomans se plurent à se constituer en

I42. Olivier Bouquet, Les pachas du sultan..., op. cit., p. 213, 344, 446.

I43. Cem Behar et Alan Duben négligent complètement l'étude des vakıf dans leur enquête sur les familles (Istanbul Households..., op. cit.). 
noblesse. Bref, les impériaux firent de la généalogie un moyen de rester ensemble et de faire savoir qu'ils continueraient de l'être, dans l'apatridie (pour les membres du hanedan), sinon dans la dispersion (pour les autres).

Dans le cas de certaines familles dont les fortunes étaient encore implantées en Turquie, les Halil Hamid Paşa-zâde par exemple, ces facteurs se conjuguèrent, au point de nourrir une passion naissante pour la généalogie. Il y eut une évolution entre les exigences du vakıf et le goût de la recherche identitaire ; il y eut un glissement, de la seule obligation de recension pour tous, au souvenir entretenu par quelques amateurs. Les descendants avaient travaillé pour les descendants à venir, en garantissant leurs droits ; ils s'intéressaient désormais aux ascendants, hors de la seule figure de l'ancêtre fondateur. De solidarités objectives et réciproques (sinon multilatérales) en appréhension subjective de soi dans une lignée, une histoire, un passé mais aussi un devenir : la généalogie faisait désormais famille. 